\title{
A kilonova as the electromagnetic counterpart to a gravitational-wave source
}

S. J. Smartt ${ }^{1}$, T.-W. Chen ${ }^{2}$, A. Jerkstrand ${ }^{3}$, M. Coughlin ${ }^{4}$, E. Kankare ${ }^{1}$, S. A. Sim ${ }^{1}$, M. Fraser ${ }^{5}$, C. Inserra $^{6}$, K. Maguire ${ }^{1}$, K. C. Chambers ${ }^{7}$, M. E. Huber ${ }^{7}$, T. Krühler ${ }^{2}$, G. Leloudas ${ }^{8}$, M. Magee ${ }^{1}$, L. J. Shingles ${ }^{1}$, K. W. Smith ${ }^{1}$, D. R. Young ${ }^{1}$, J. Tonry ${ }^{7}$, R. Kotak ${ }^{1}$, A. Gal-Yam ${ }^{9}$, J. D. Lyman ${ }^{10}$, D. S. Homan ${ }^{11}$, C. Agliozzo ${ }^{12,13}$, J. P. Anderson ${ }^{14}$, C. R. Angus ${ }^{6}$, C. Ashall ${ }^{15}$, C. Barbarino ${ }^{16}$, F. E. Bauer ${ }^{13,17,18}$, M. Berton ${ }^{19,20}$, M. T. Botticella ${ }^{21}$, M. Bulla ${ }^{63}$, J. Bulger ${ }^{7}$, G. Cannizzaro ${ }^{22,41}$, Z. Cano ${ }^{23}$, R. Cartier ${ }^{6}$, A. Cikota ${ }^{24}$, P. Clark ${ }^{1}$, A. De Cia ${ }^{24}$, M. Della Valle ${ }^{21,25}$, L. Denneau ${ }^{7}$, M. Dennefeld ${ }^{26}$, L. Dessart ${ }^{27}$, G. Dimitriadis ${ }^{6}$, N. Elias-Rosa ${ }^{28}$, R. E. Firth ${ }^{6}$, H. Flewelling ${ }^{7}$, A. Flörs $^{3,24,29}$, A. Franckowiak ${ }^{30}$, C. Frohmaier ${ }^{31}$, L. Galbany ${ }^{32}$, S. González-Gaitán ${ }^{33}$, J. Greiner ${ }^{2}$, M. Gromadzki ${ }^{34}$, A. Nicuesa Guelbenzu ${ }^{35}$, C. P. Gutiérrez ${ }^{6}$, A. Hamanowicz ${ }^{24,34}$, L. Hanlon ${ }^{5}$, J.

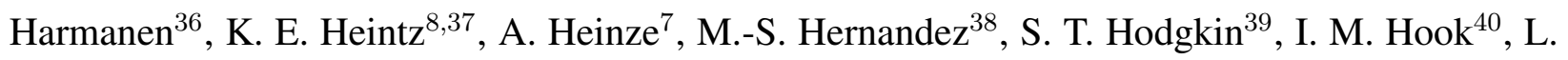
Izzo $^{23}$, P. A. James ${ }^{15}$, P. G. Jonker ${ }^{22,41}$, W. E. Kerzendorf ${ }^{24}$, S. Klose ${ }^{35}$, Z. Kostrzewa-Rutkowska ${ }^{22,41}$,

M. Kowalski ${ }^{30,42}$, M. Kromer ${ }^{43,44}$, H. Kuncarayakti ${ }^{36,45}$, A. Lawrence ${ }^{11}$, T. B. Lowe ${ }^{7}$, E. A. Magnier ${ }^{7}$, I. Manulis ${ }^{9}$, A. Martin-Carrillo ${ }^{5}$, S. Mattila ${ }^{36}$, O. McBrien ${ }^{1}$, A. Müller ${ }^{46}$, J. Nordin ${ }^{42}$, D. O’Neill ${ }^{1}$, F. Onori ${ }^{22,41}$, J. T. Palmerio ${ }^{47}$, A. Pastorello ${ }^{48}$, F. Patat ${ }^{24}$, G. Pignata ${ }^{12,13}$, Ph. Podsiadlowski ${ }^{49}$, M.

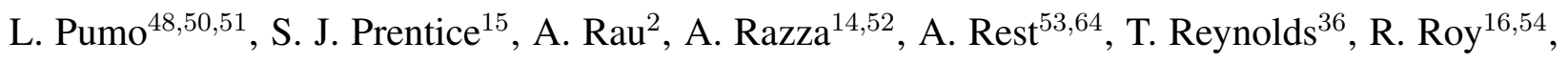
A. J. Ruiter ${ }^{55,56,57}$, K. A. Rybicki ${ }^{34}$, L. Salmon ${ }^{5}$, P. Schady ${ }^{2}$, A. S. B. Schultz 7 , T. Schweyer ${ }^{2}$, I. R. Seitenzahl ${ }^{55,56}$, M. Smith ${ }^{6}$, J. Sollerman ${ }^{16}$, B. Stalder ${ }^{58}$, C. W. Stubbs ${ }^{59}$, M. Sullivan ${ }^{6}$, H.

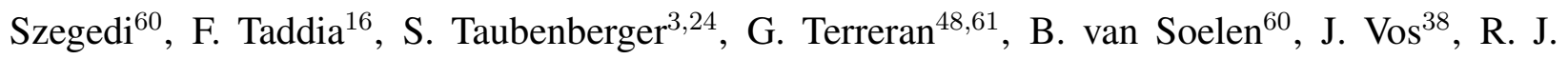

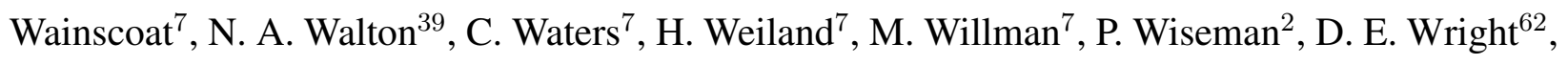


Ł. Wyrzykowski ${ }^{34} \&$ O. Yaron ${ }^{9}$

${ }^{1}$ Astrophysics Research Centre, School of Mathematics and Physics, Queens University Belfast, Belfast BT7 1NN, UK

${ }^{2}$ Max-Planck-Institut für Extraterrestrische Physik, Giessenbach-Str. 1, D-85748, Garching, Munich, Germany

${ }^{3}$ Max-Planck Institut für Astrophysik, Karl-Schwarzschild-Str. 1, D-85748 Garching, Munich, Germany

${ }^{4}$ LIGO Laboratory West Bridge, Rm. 257 California Institute of Technology, MC 100-36, Pasadena, CA 91125

${ }^{5}$ School of Physics, O’Brien Centre for Science North, University College Dublin, Belfield, Dublin 4, Ireland

${ }^{6}$ Department of Physics and Astronomy, University of Southampton, Southampton, Hampshire SO17 1BJ, UK

${ }^{7}$ Institute for Astronomy, University of Hawaii, 2680 Woodlawn Drive, Honolulu, Hawaii 96822, USA

${ }^{8}$ Dark Cosmology Centre, Niels Bohr Institute, University of Copenhagen, Juliane Maries Vej 30, 2100 Copenhagen Ø, Denmark

${ }^{9}$ Department of Particle Physics and Astrophysics, Weizmann Institute of Science, Rehovot 76100, Israel

${ }^{10}$ Department of Physics, University of Warwick, Coventry CV4 7AL, UK

${ }^{11}$ Institute for Astronomy, SUPA (Scottish Universities Physics Alliance), University of Edinburgh, 
Royal Observatory, Blackford Hill, Edinburgh EH9 3HJ, UK

${ }^{12}$ Departamento de Ciencias Fisicas, Universidad Andres Bello, Avda. Republica 252, Santiago, Chile

${ }^{13}$ Millennium Institute of Astrophysics (MAS), Nuncio Monseñor Sótero Sanz 100, Providencia, Santiago, Chile

${ }^{14}$ European Southern Observatory, Alonso de Córdova 3107, Casilla 19, Santiago, Chile

${ }^{15}$ Astrophysics Research Institute, Liverpool John Moores University, IC2, Liverpool Science Park, 146 Brownlow Hill, Liverpool L3 5RF, UK

${ }^{16}$ The Oskar Klein Centre, Department of Astronomy, Stockholm University, AlbaNova, 10691 Stockholm, Sweden

${ }^{17}$ Instituto de Astrofísica and Centro de Astroingeniería, Facultad de Física, Pontificia Universidad Católica de Chile, Casilla 306, Santiago 22, Chile

${ }^{18}$ Space Science Institute, 4750 Walnut Street, Suite 205, Boulder, Colorado 80301

${ }^{19}$ Dipartimento di Fisica e Astronomia “G. Galilei”, Università di Padova, Vicolo dell'Osservatorio 3, 35122, Padova, Italy

${ }^{20}$ INAF - Osservatorio Astronomico di Brera, via E. Bianchi 46, 23807 Merate (LC), Italy

${ }^{21}$ INAF - Osservatorio Astronomico di Capodimonte, via Salita Moiariello 16, 80131 Napoli, Italy

${ }^{22}$ SRON, Netherlands Institute for Space Research, Sorbonnelaan 2, NL-3584 CA Utrecht, The Netherlands

${ }^{23}$ Instituto de Astrofísica de Andalucía (IAA-CSIC), Glorieta de la Astronomía s/n, E-18008, Granada, Spain 
${ }^{24}$ European Southern Observatory, Karl-Schwarzschild Str. 2, 85748 Garching bei München, Germany

${ }^{25}$ ICRANet-Pescara, Piazza della Repubblica 10, I-65122 Pescara, Italy

${ }^{26}$ IAP/CNRS and University Pierre et Marie Curie, Paris, France

${ }^{27}$ Unidad Mixta Internacional Franco-Chilena de Astronomía (CNRS UMI 3386), Departamento de Astronomía, Universidad de Chile, Camino El Observatorio 1515, Las Condes, Santiago, Chile ${ }^{28}$ Istituto Nazionale di Astrofisica, Viale del Parco Mellini 84, Roma I-00136, Italy

${ }^{29}$ Physik-Department, Technische Universität München, James-Franck-Straße 1, 85748 Garching bei München

${ }^{30}$ Deutsches Elektronen Synchrotron DESY, D-15738 Zeuthen, Germany

${ }^{31}$ Institute of Cosmology and Gravitation, Dennis Sciama Building, University of Portsmouth, Burnaby Road, Portsmouth PO1 3FX, UK

${ }^{32}$ PITT PACC, Department of Physics and Astronomy, University of Pittsburgh, Pittsburgh, PA 15260, USA

${ }^{33}$ CENTRA, Instituto Superior Técnico - Universidade de Lisboa, Portugal

${ }^{34}$ Warsaw University Astronomical Observatory, Al. Ujazdowskie 4, 00-478 Warszawa, Poland

${ }^{35}$ Thüringer Landessternwarte Tautenburg, Sternwarte 5, 07778 Tautenburg, Germany

${ }^{36}$ Tuorla observatory, Department of Physics and Astronomy, University of Turku, Väisäläntie 20, FI-21500 Piikkiö, Finland

${ }^{37}$ Centre for Astrophysics and Cosmology, Science Institute, University of Iceland, Dunhagi 5, 107 Reykjavík, Iceland 
${ }^{38}$ Instituto de Física y Astronomía, Universidad de Valparaiso, Gran Bretaña 1111, Playa Ancha, Valparaíso 2360102, Chile

${ }^{39}$ Institute of Astronomy, University of Cambridge, Madingley Road, Cambridge CB3 OHA, UK

${ }^{40}$ Department of Physics, Lancaster University, Lancaster LA1 4YB, UK

${ }^{41}$ Department of Astrophysics/IMAPP, Radboud University, P.O. Box 9010, NL-6500 GL Nijmegen, The Netherlands

${ }^{42}$ Institut fur Physik, Humboldt-Universitat zu Berlin, Newtonstr. 15, D-12489 Berlin, Germany

${ }^{43}$ Zentrum für Astronomie der Universität Heidelberg, Institut für Theoretische Astrophysik, Philosophenweg 12, 69120 Heidelberg, Germany

${ }^{44}$ Heidelberger Institut für Theoretische Studien, Schloss-Wolfsbrunnenweg 35, 69118 Heidelberg, Germany

${ }^{45}$ Finnish Centre for Astronomy with ESO (FINCA), University of Turku, Väisäläntie 20, 21500 Piikkiö, Finland

${ }^{46}$ Max Planck Institute for Astronomy, Königstuhl 17, 69117 Heidelberg, Germany

${ }^{47}$ Sorbonne Universités, UPMC Univ. Paris 6 and CNRS, UMR 7095, Institut dAstrophysique de Paris, 98 bis bd Arago, 75014 Paris, France

${ }^{48}$ INAF-Osservatorio Astronomico di Padova, Vicolo dell'Osservatorio 5, 35122 Padova, Italy

${ }^{49}$ Department of Astrophysics, University of Oxford, Oxford, OX1 3RH, UK

${ }^{50}$ Università degli studi di Catania, DFA DIEEI, Via Santa Sofia 64, 95123 Catania, Italy

${ }^{51}$ INFN-Laboratori Nazionali del Sud, Via Santa Sofia 62, Catania, 95123, Italy

${ }^{52}$ Department of Astronomy, Universidad de Chile, Camino El Observatorio 1515, Las Condes, 
Santiago de Chile, Chile

${ }^{53}$ Space Telescope Science Institute, 3700 San Martin Drive, Baltimore, MD 21218, USA

${ }^{54}$ Inter-University Centre for Astronomy and Astrophysics (IUCAA), Pune - 411007, India

${ }^{55}$ School of Physical, Environmental, and Mathematical Sciences, University of New South Wales, Australian Defence Force Academy, Canberra, ACT 2600, Australia

${ }^{56}$ Research School of Astronomy and Astrophysics, The Australian National University, Canberra, ACT 2611, Australia

${ }^{57}$ ARC Centre of Excellence for All-sky Astrophysics (CAASTRO)

${ }^{58}$ LSST, 950 N. Cherry Ave, Tucson, AZ, 85719

${ }^{59}$ Department of Physics, Harvard University, Cambridge, MA 02138, USA

${ }^{60}$ Department of Physics, University of the Free State, Bloemfontein, 9300 South Africa

${ }^{61}$ Center for Interdisciplinary Exploration and Research in Astrophysics (CIERA) and Department of Physics and Astronomy, Northwestern University, Evanston, IL 60208

${ }^{62}$ School of Physics and Astronomy, University of Minnesota, 116 Church Street SE, Minneapolis, MN 55455-0149

${ }^{63}$ The Oskar Klein Centre, Department of Physics, Stockholm University, AlbaNova, 10691 Stockholm, Sweden.

${ }^{64}$ Department of Physics and Astronomy, The Johns Hopkins University, 3400 North Charles Street, Baltimore, MD 21218, USA

Gravitational waves were discovered with the detection of binary black hole mergers ${ }^{1}$ and they should also be detectable from lower mass neutron star mergers. These are predicted 
to eject material rich in heavy radioactive isotopes that can power an electromagnetic signal called a kilonova $2,4,5$. The gravitational wave source GW170817 arose from a binary neutron star merger in the nearby Universe with a relatively well confined sky position and distance estimate ${ }^{6}$. Here we report observations and physical modelling of a rapidly fading electromagnetic transient in the galaxy NGC4993, which is spatially coincident with GW170817 and a weak short gamma-ray burst ${ }^{7}$, . The transient has physical parameters broadly matching the theoretical predictions of blue kilonovae from neutron star mergers. The emitted electromagnetic radiation can be explained with an ejected mass of $0.04 \pm 0.01 \mathbf{M}_{\odot}$ with an opacity of $\kappa \leq 0.5 \mathbf{c m}^{2} \mathbf{g}^{-1}$ at a velocity of $0.2 \pm 0.1 \mathrm{c}$. The power source is constrained to have a power law slope of $\beta=-1.2_{-0.3}^{+0.3}$, consistent with radioactive powering from r-process nuclides. We identify line features in the spectra that are consistent with light r-process elements $(90<\mathrm{A}<140)$. As it fades, the transient rapidly becomes red, and emission may have contribution by a higher opacity, lanthanide-rich ejecta component. This indicates that neutron star mergers produce gravitational waves, radioactively powered kilonovae, and are a nucleosynthetic source of the r-process elements.

The Advanced LIGO and Advanced Virgo experiments ${ }^{9}[0$ detected gravitational wave emission (called GW170817) on 2017 Aug 17 12:41:04 UT (MJD 57982.528524) ${ }^{6}$ from the merger of two in-spiralling objects consistent with being a neutron star binary. The source and initial skymap was announced to the collaborating follow-up groups at 2017 Aug 17 13:08:17 UT. The small sky area of 33.6 square degrees of the $90 \%$ probability contour in the combined LIGO and Virgo analysis (in the LALInference map ${ }^{11}[12$ ) prompted us to plan to tile the region with our Pan-STARRS 
program to search for electromagnetic counterparts of gravitational wave sources. However, given the low elevation and report of a transient discover ${ }^{13}$ in a galaxy within the volume constrained by LIGO-Virgo (released at 2017 Aug 18 01:05:23 UT) ${ }^{13}$, we changed strategy to gather early multi-colour photometry of the source called SSS17 $\mathrm{a}^{13}$ and DLT17ck $\mathrm{k}^{114}$ by the two teams, and formally registered with an IAU name of AT2017gfo. We began imaging the source at 2017 Aug 18 05:33 UT with Pan-STARRS1 and then took our first spectrum under the extended Public ESO Spectroscopic Survey for Transient Objects (ePESSTO ${ }^{15}$ ) on 2017 Aug 18 23:20 UT. We started photometric monitoring with GROND on 2017 Aug 18 23:15 UT providing combined photometry across the optical and infra-red bands, $\operatorname{UgrizJ} H K_{\mathrm{s}}$ (Figure1 1 and Extended Data Figure 1 and Methods).

Before GW170817, we had monitored this sky area with ATLAS ${ }^{16}$ between 2015 Dec 12 15:50 UT and 2017 Aug 01 06:19 UT observing a total of 414 images, typically with 4-5 images per night. No transient or astrophysical variability was detected at the position in ATLAS difference images to $5 \sigma$ limits of $o=18.7$ and $c=19.3 \mathrm{mag}$ (see Methods and Extended Data Figure2). The ATLAS pre-discovery limits show that it is unlikely that AT2017gfo is a transient in NGC4993 which is not physically associated with GW170817 and is merely a chance coincidence. We assume that AT2017gfo is an unusual, supernova-like explosion in NGC4993 that exploded within 16 days of GW170817. The number of supernovae expected within the four-dimensional space (volume and time) defined by the LIGO distance range for GW170817, $(73 \mathrm{Mpc})$ and within the refined $90 \%$ sky area of 28 square degrees (reduced in the final released map ${ }^{6}$ ), and within 16 days is $n_{\mathrm{SN}}=0.005$, assuming a supernova rate ${ }^{17}$ of $R_{\mathrm{SN}}=1.0 \times 10^{-4} \mathrm{Mpc}^{-3} \mathrm{yr}^{-1}$. It is unlike any 
known nearby, or distant, supernova (see Extended Data Figures 1 and 3). If we assume that the rate of events similar to AT2017gfo is $\sim 1 \%$ of the volumetric supernova rate (see Methods Section) then the probability of a chance coincidence in space and time is $p=5 \times 10^{-5}$ (equivalent to $4 \sigma$ significance).

We calculated a bolometric lightcurve and total luminosity emitted assuming a distance to NGC4993 of $d=40 \pm 4 \mathrm{Mpc}$ and appropriate Galactic foreground extinction (see Methods section for details of the calculation). In Figure 2 we compare the absolute magnitude of AT2017gfo in all bands to several kilonova models calculated for NS-NS mergers predicted before this discovery. All models are powered by radioactivity of r-process elements $(\beta \text {-decays, } \alpha \text {-decays and fission })^{2}$ formed in the merger. The set includes both simple and advanced radiative-transfer treatments, and they differ in their treatment of the opacity of the ejected material. Each of the models predict fast-fading red transients, with some variation in luminosity and decline rate. If heavy lanthanides (atomic masses $A>140$ ) dominate the ejecta then the opacity is predicted to be high ${ }^{\sqrt[4]{4}}$, with the inevitable consequence of a longer duration, infra-red transient as seen in Figure 2 for the Barnes et al.$^{18}$ and the Tanaka \& Hotokezaka ${ }^{4}$ lanthanide rich models. These models do reproduce the near infra-red luminosity at 7 - 14 days but the observed early emission which is hot and blue is not reproduced in merger models which are dominated by heavy lanthanide composition. The Metzger model 19 can produce a "blue kilonova" by using a lower opacity, appropriate for light r-process elements (a blend of elements with $90<A<140$ ). This model has a grey opacity and a thermalization efficiency ${ }^{20}$ is assumed. The slope of the ejecta velocity distribution $\alpha$ is defined such that the amount of mass travelling above velocity $v$ scales as $M(>v)=M_{0}\left(v / v_{0}\right)^{-\alpha}$. This 
gives a good fit to the data, suggesting that very high opacities, which block much of the optical light are not applicable in the first $3-4$ days or depend on orientation ${ }^{19}$. A minimum velocity value $v_{e j} \simeq 0.1 \mathrm{c}$ is preferred, which within current simulation uncertainties is similar to both dynamic and wind ejecta ${ }^{20}$. We assess this is more likely a wind component as these can more easily obtain low opacity (see Methods Section).

We further explore the "blue kilonova" scenario by calculating our own quantitative models based on the semi-analytic methods of Arnett ${ }^{[21}$, extended to adopt a general term for powering $\mathrm{g}^{22[23]}$ and Metzger ${ }^{19}$. For the Arnett model, we used a power law for the power term with absolute scaling (decay per energy per gram per second) after 1 day as obtained in radioactivity models ${ }^{19}$ with a free exponent $\beta$ (such that $P \propto t^{\beta}$ ). The other parameters are ejected mass $M_{\mathrm{ej}}$, energy $E$ (or equivalently velocity $v_{e j}$ as defined by $\frac{1}{2} M_{e j} v_{e j}^{2}=E$ ) and opacity $\kappa$. As $\kappa$ and $E$ are fully degenerate (as $\kappa / \sqrt{E}$ ) when trapping is not explicitly coupled (as here), we effectively fit over $M_{e j}, \beta$ and $\kappa / v_{e j}$. The best fits are shown in Figure 3 . With no other constraints except that we enforce $v_{e j}<0.2 \mathrm{c}$, the best fitting models have $M_{\mathrm{ej}}=0.02 \pm 0.01 \mathrm{M}_{\odot}, \kappa=0.1 \times\left(\frac{v_{e j}}{0.2 c}\right) \mathrm{cm}^{2} \mathrm{~g}^{-1}$ and $\beta=-1.5_{-0.2}^{+0.3}$. If we also implement a thermalization efficiency ${ }^{20}, 19$ to account for efficiency of the powering mechanism to provide heat to the ejecta, the values change to $M_{\mathrm{ej}}=0.04 \pm 0.01 \mathrm{M}_{\odot}$, $\kappa=0.1 \times\left(\frac{v_{e j}}{0.2 c}\right) \mathrm{cm}^{2} \mathrm{~g}^{-1}$ and $\beta=-1.2_{-0.3}^{+0.3}$ (see Extended Data Figures 45 for probability density plots of the parameters).

The mass and power law exponent are remarkably close to predicted kilonova values. In particular, $\beta$ has been shown to be robustly between -1.3 and -1.2 for r-process radioactivity, 
with weak sensitivity to electron fraction and thermodynamic trajectory 24 .25]. We find the data can be explained with a low mass of ejecta having opacity consistent with a blend of elements in the $90<A<140$ mass range powered by r-process radioactive decays. Our models interpret the first three data points as the end of the diffusion phase, and match the later points with the early tail phase (starting at $2-3$ days).

Many previous kilonova models predict that if heavy r-process elements such as the lanthanides and actinides are produced then high opacities of around $\kappa=10 \mathrm{~cm}^{2} \mathrm{~g}^{-1}$ would be likely ${ }^{420}$. In Figure 3 we show the best fits forcing $\kappa=10 \mathrm{~cm}^{2} \mathrm{~g}^{-1}$. No model with this high opacity is able to fit all the data points well, but it can fit the later data points. In these high-opacity models all observations are still within the diffusion phase, but a steeper power law for energy input ( $\beta \simeq-2$ ) is favoured to produce the right emergent luminosity, no longer consistent with $t^{-1.3}$. If our reconstructed bolometric light curve is accurate at all epochs, there is not much room for a second component at later times as the blue one cannot drop faster than the power source term. However, its possible that 2-component SED fitting would give somewhat different late time bolometric estimates. Then a two-component model where the early light curve is produced by a low-opacity ejecta (a wind component), and the later by a high-opacity one (dynamic ejecta) could also be possible. The early blue flux is unlikely to be from a relativistic jet ${ }^{26}$ and an afterglow from the weak gamma ray signal that was detected ${ }^{7} \mid \frac{8}{8}$, due to the rapid reddening and cooling and the X-ray non-detections.

The optical and NIR spectra support the ejecta being dominated by the light r-process ele- 
ments at least at early stages. We have used the TARDIS code ${ }^{27}$ to construct simple models to guide interpretation of our spectra. Our earliest NTT spectrum (epoch +1.4 day) is fairly well parameterised by a black-body of $T_{\text {eff }}=5200 \mathrm{~K}$, and does not show the prominent spectral features ( $\mathrm{Ca}, \mathrm{Mg}$ or $\mathrm{Si}$ ) commonly detected in normal supernova spectra (see Extended Data Figure 3 ). There are two broad and blended structures at $7400 \AA$ and $8300 \AA$, respectively, which become stronger in the subsequent spectra. We have extended the TARDIS atomic database to include lines of elements with atomic number $31<Z<60$ (or $60<A<140$ ) from the Kurucz atomic line list ${ }^{28}$, although the available atomic data for these heavy elements is of limited quality and quantity. We propose the broad feature at 7000-7500 $\AA$ is from neutral Caesium $(A=133)$, and is the $6 \mathrm{~s}^{2} \mathrm{~S} \rightarrow 6 \mathrm{p}{ }^{2} \mathrm{P}$ resonance doublet $(\lambda \lambda 8521,8943)$ at a photospheric velocity of $\sim 0.15-0.20 \mathrm{c}$ (see Figure 4). Our model predicts no other strong features of Cs I in the observed region, which could be used to confirm (or refute) this identification. For the redder absorption, we can identify an intriguing potential match with the Tellurium $(A=128) 5 \mathrm{p}^{3}\left({ }^{4} \mathrm{~S}\right) 6 \mathrm{~s}{ }^{5} \mathrm{~S} \rightarrow 5 \mathrm{p}^{3}\left({ }^{4} \mathrm{~S}\right) 6 \mathrm{p}{ }^{5} \mathrm{P}$ triplet of Te I. This moderate-excitation multiplet could plausibly be excited at the temperature in our model and would produce absorption around 8000-8500 $\AA$. Reliable oscillator strengths for this multiplet are not available in the NIST atomic spectra database $\mathrm{e}^{29}$, but we included it in our TARDIS spectral model by adopting $\log g f=0$ for each member of the triplet. This illustrates a broadly consistent match with the velocity and thermal conditions that correspond to the Cs I identification. The ionized states (Cs II and Te II) are predicted to dominate by mass, meaning that our model can not provide reliable elemental mass estimates (see Methods Section for more details). The second spectrum covering 0.35-2.2 $\mu \mathrm{m}$ further indicates that Cs I and Te I are plausible candidates. The 
photospheric velocity adopted in TARDIS $(0.2 \mathrm{c}$ for the $+1.4 \mathrm{~d}$ spectrum) is roughly consistent with that used in our light-curve model at this phase. We further checked atomic data line-lists for possible light r-process elements ${ }^{30}$ in this range, finding neutral and singly ionized $\mathrm{Sb}$, I and Xe transitions. The Xe I lines align well with possible absorption features seen around 1.48 and $1.75 \mu \mathrm{m}$ in our +4.4 day spectrum, along with Cs I and Te I features. However, in our TARDIS models, the excitation energies of the relevant Xe I states are too high to make lines of this ion an important contributor at the temperatures considered, unless it is non-thermally excited.

The light curve and spectra of this fast-fading transient are consistent with an ejecta being high velocity, low mass, and powered by a source consistent with the r-process decay timescales. We can fit the full lightcurve with relatively low-opacity material consistent with the light r-process elements. We can't rule out that a second component consisting of the heavy lanthanides and actinides contributes to the infra-red flux after 3 days. Orientation effects of the dynamic ejecta and wind may play a role in what is observed ${ }^{19}$. This shows that the nucleosynthetic origin of the r-process elements ${ }^{\sqrt{31}}$ can be in neutron star mergers. 
1. Abbott, B. P. et al. Observation of Gravitational Waves from a Binary Black Hole Merger. Physical Review Letters 116, 061102 (2016). 1602.03837.

2. Metzger, B. D. et al. Electromagnetic counterparts of compact object mergers powered by the radioactive decay of r-process nuclei. Mon. Not. R. Astron. Soc. 406, 2650-2662 (2010). 1001.5029 .

3. Kasen, D., Badnell, N. R. \& Barnes, J. Opacities and Spectra of the r-process Ejecta from Neutron Star Mergers. Astrophys. J. 774, 25 (2013). 1303.5788.

4. Tanaka, M. \& Hotokezaka, K. Radiative Transfer Simulations of Neutron Star Merger Ejecta. Astrophys. J. 775, 113 (2013). 1306.3742.

5. Rosswog, S. et al. Detectability of compact binary merger macronovae. Classical and Quantum Gravity 34, 104001 (2017). 1611.09822.

6. Abbott, B. P. et al. GW170817: observation of gravitational waves from a binary neutron star inspiral. Physical Review Letters 116, 161101 (2017).

7. Goldstein, A. et al. An ordinary short gamma-ray burst with extraordinary implications: Fermi-GBM detection of GRB 170817A.. Astrophysical Journal Letters 848, https://doi.org/10.3847/2041-8213/aa8f41 (2017).

8. Savchenko, V. et al. INTEGRAL detection of the rst prompt gamma-ray signal coincident with the gravitational event GW170817. Astrophysical Journal Letters 848, https://doi.org/10.3847/2041-8213/aa8f94 (2017). 
9. Aasi, J. et al. Characterization of the LIGO detectors during their sixth science run. Classical and Quantum Gravity 32, 115012 (2015). 1410.7764.

10. Acernese, F. et al. Advanced Virgo: a second-generation interferometric gravitational wave detector. Classical and Quantum Gravity 32, 024001 (2015). 1408.3978.

11. Veitch, J. et al. Parameter estimation for compact binaries with ground-based gravitationalwave observations using the LALInference software library. Phys.R. D 91, 042003 (2015). 1409.7215 .

12. The LIGO Scientific Collaboration and the Virgo Collaboration. 21527. GRB Coordinates Network 21527 (2017).

13. Coulter, D.A. et al. Swope supernova survey 2017a (SSS17a), the optical counterpart to a gravitational wave source.. Science 848, http://doi.org/10.1126/ science.aap9811 (2017).

14. Valenti, S. et al. The discovery of the electromagnetic counterpart of GW170817: kilonova AT2017gfo/DLT17ck. Astrophysical Journal Letters 848, http://doi. org/10.3847/20418213/aa8edf (2017).

15. Smartt, S. J. et al. PESSTO: survey description and products from the first data release by the Public ESO Spectroscopic Survey of Transient Objects. Astron. Astrophys. 579, A40 (2015). 1411.0299

16. Stalder, B. et al. Observations of the GRB afterglow ATLAS17aeu and its possible association with GW170104. ArXiv e-prints (2017). 1706.00175. 
17. Li, W. et al. Nearby supernova rates from the Lick Observatory Supernova Search - III. The rate-size relation, and the rates as a function of galaxy Hubble type and colour. Mon. Not. R. Astron. Soc. 412, 1473-1507 (2011). 1006.4613.

18. Barnes, J., Kasen, D., Wu, M.-R. \& Martínez-Pinedo, G. Radioactivity and Thermalization in the Ejecta of Compact Object Mergers and Their Impact on Kilonova Light Curves. Astrophys. J. 829, 110 (2016). 1605.07218.

19. Metzger, B. D. Kilonovae. Living Reviews in Relativity 20, 3 (2017). 1610.09381.

20. Barnes, J. \& Kasen, D. Effect of a High Opacity on the Light Curves of Radioactively Powered Transients from Compact Object Mergers. Astrophys. J. 775, 18 (2013). 1303.5787.

21. Arnett, W. D. Type I supernovae. I - Analytic solutions for the early part of the light curve. Astrophys. J. 253, 785-797 (1982).

22. Inserra, C. et al. Super-luminous Type Ic Supernovae: Catching a Magnetar by the Tail. Astrophys. J. 770, 128 (2013). 1304.3320.

23. Chatzopoulos, E., Wheeler, J. C. \& Vinko, J. Generalized Semi-analytical Models of Supernova Light Curves. Astrophys. J. 746, 121 (2012). 1111.5237.

24. Korobkin, O., Rosswog, S., Arcones, A. \& Winteler, C. On the astrophysical robustness of the neutron star merger r-process. Mon. Not. R. Astron. Soc. 426, 1940-1949 (2012). 1206. 2379. 
25. Wanajo, S. et al. Production of All the r-process Nuclides in the Dynamical Ejecta of Neutron Star Mergers. Astrophys. J. Letters 789, L39 (2014). 1402.7317.

26. Tanvir, N. R. et al. A 'kilonova' associated with the short-duration $\gamma$-ray burst GRB 130603B. Nature 500, 547-549 (2013). 1306.4971.

27. Kerzendorf, W. E. \& Sim, S. A. A spectral synthesis code for rapid modelling of supernovae. Mon. Not. R. Astron. Soc. 440, 387-404 (2014). 1401.5469.

28. Kurucz, R. \& Bell, B. Atomic Line Data. Atomic Line Data (R.L. Kurucz and B. Bell) Kurucz CD-ROM No. 23. Cambridge, Mass.: Smithsonian Astrophysical Observatory, 23 (1995).

29. Kramida, A., Yu. Ralchenko, Reader, J. \& and NIST ASD Team. NIST Atomic Spectra Database (ver. 5.3), [Online]. Available: http://physics.nist.gov/asd [2017, September 14]. National Institute of Standards and Technology, Gaithersburg, MD. (2015).

30. Burris, D. L. et al. Neutron-Capture Elements in the Early Galaxy: Insights from a Large Sample of Metal-poor Giants. Astrophys. J. 544, 302-319 (2000). a stro-ph/ 0005188.

31. Burbidge, E. M., Burbidge, G. R., Fowler, W. A. \& Hoyle, F. Synthesis of the Elements in Stars. Reviews of Modern Physics 29, 547-650 (1957).

32. Kasen, D., Fernández, R. \& Metzger, B. D. Kilonova light curves from the disc wind outflows of compact object mergers. Mon. Not. R. Astron. Soc. 450, 1777-1786 (2015). 1411.3726.

33. Wollaeger, R. T. et al. Impact of ejecta morphology and composition on the electromagnetic signatures of neutron star mergers. ArXiv e-prints (2017). 1705.07084. 
Author Contributions SJS is PI of ePESSTO, co-lead for Pan-STARRS GW follow-up, led writing of text and managed project. AJ wrote the lightcurve fitting code, led the modelling and co-wrote the text. MC provided code for modelling and MCMC, provided analysis and text, and KW provided input. SAS, LJS and MM did the TARDIS modelling, assisted by AG-Y in line identification. T-WC, JG, SK, AR, PS, TS, TK, PW and ANG managed, executed, reduced and provided GROND data. EK, MF, CI, KM, TK and GL reduced and analysed photometry and spectra and contributed to analysis, text and figures. KWS and DRY ran data management for ATLAS and Pan-STARRS analysis. JT is ATLAS lead and provided data. KCC is Pan-STARRS director, co-lead of GW follow-up and managed the observing sequences. Pan-STARRS and ATLAS data and products were provided by the team of MEH, JB, LD, HF, TBL, EAM, AR, ASBS, BS, RJW, CW, HW, MW and DEW. CWS is ATLAS co-lead for GW follow-up and contributed to text. OMB and PC checked the ATLAS data for candidates and OMB provided manuscript editing support. The ePESSTO project was delivered by the following, who have contributed to data, analysis and text comments: RK, JDL, DSH, CA, JPA, CRA, CA, CB, FEB, MB, MB, ZC, RC, AC, PC, AdC, MTB, MDV, MD, GD, NE-R, REF, AF, AF, CF, LB, SG-G, MG, CPG, AH, JH, KEH, AH, M-SH, STH, IMH, LI, PAJ, PGJ, ZK-R, MK, MK, HK, AL, IM, SM, JN, DON, FO, JTP, AP, FP, GP, MLP, SJP, TR, RR, AJR, KAR, IRS, MS, JS, MS, FT, ST, GT, JV, NAW, ŁW, OY, GC and AR. PP provided text and analysis comments. The 1.5B telescope data were provided, reduced and analysed by LH, AM-C, LS, HS and BvS. AM reduced and analysed the NACO and VISIR data.

\section{Author Information}

Correspondence Correspondence and requests for materials should be addressed to S. J. Smartt (email: s.smartt@qub.ac.uk) 
Competing Interests The authors declare that they have no competing financial interests. 

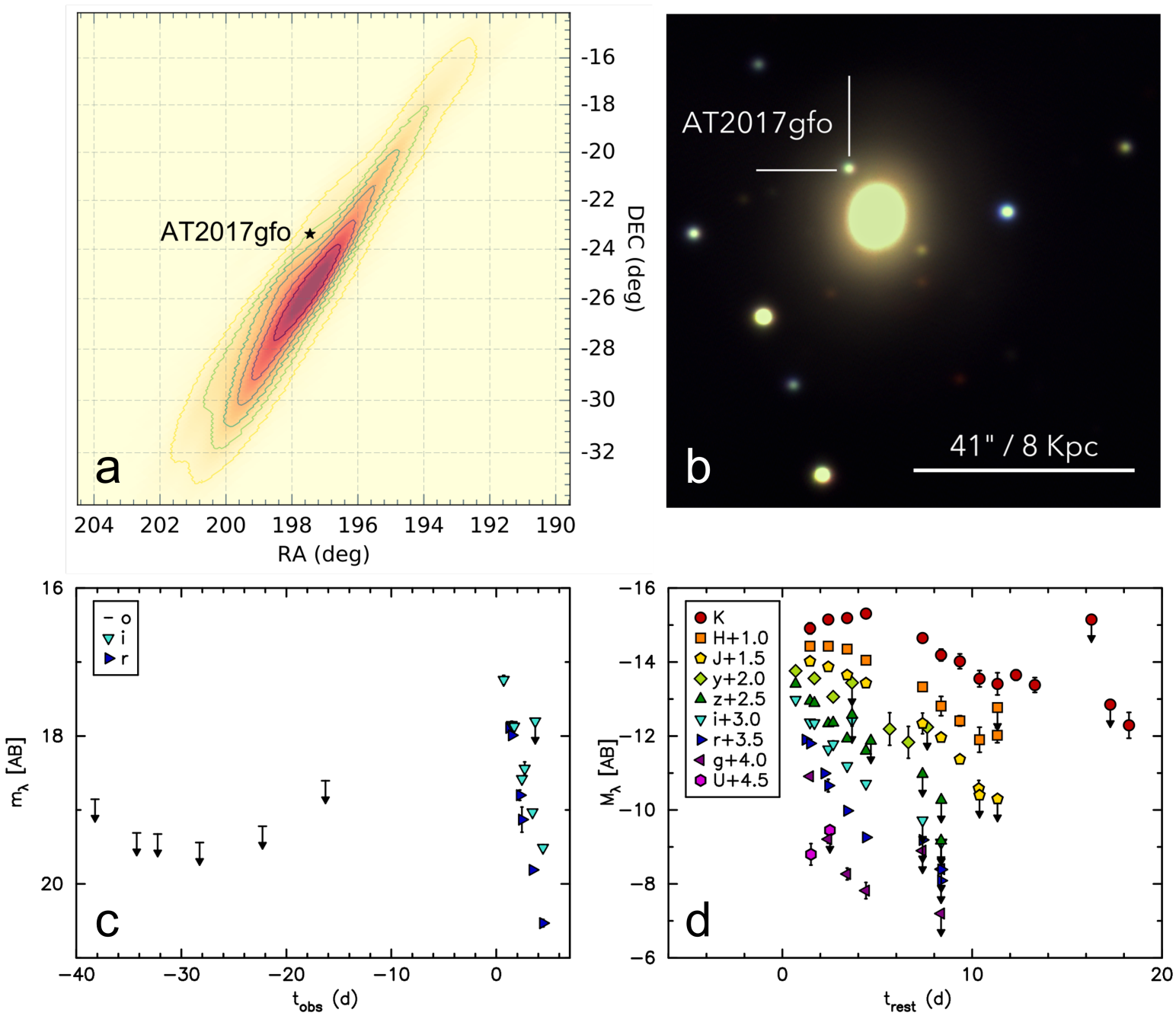

Figure 1: | Observational data summary a: The position of AT2017gfo lying within the Ligo-Virgo skymap ${ }^{11} 6$ b: Color composite image of AT2017gfo from GROND on 2017 Aug 18 (MJD 57983.969, 1.44 days after GW170817 discovery. The transient is 8.50" North, 5.40" East of the centre of NGC4993, an S0 galaxy at a distance of $40 \pm 4 \mathrm{Mpc}$. This is a projected distance of $2 \mathrm{kpc}$. The source is measured at position of RA=13:09:48.08 DEC=-23:22:53.2 J2000 $\left( \pm 0.1^{\prime \prime}\right.$ in each $)$ in our Pan-STARRS1 images. c: ATLAS limits between 40 and 16 days before discovery (orange filter), plus the Pan-STARRS1 and GROND $r$ and $i$-band light curve. d: Our full light curve data, which provides a reliable bolometric light curve for analysis. Upper limits are $3 \sigma$ and uncertainties on the measured points are $1 \sigma$. 


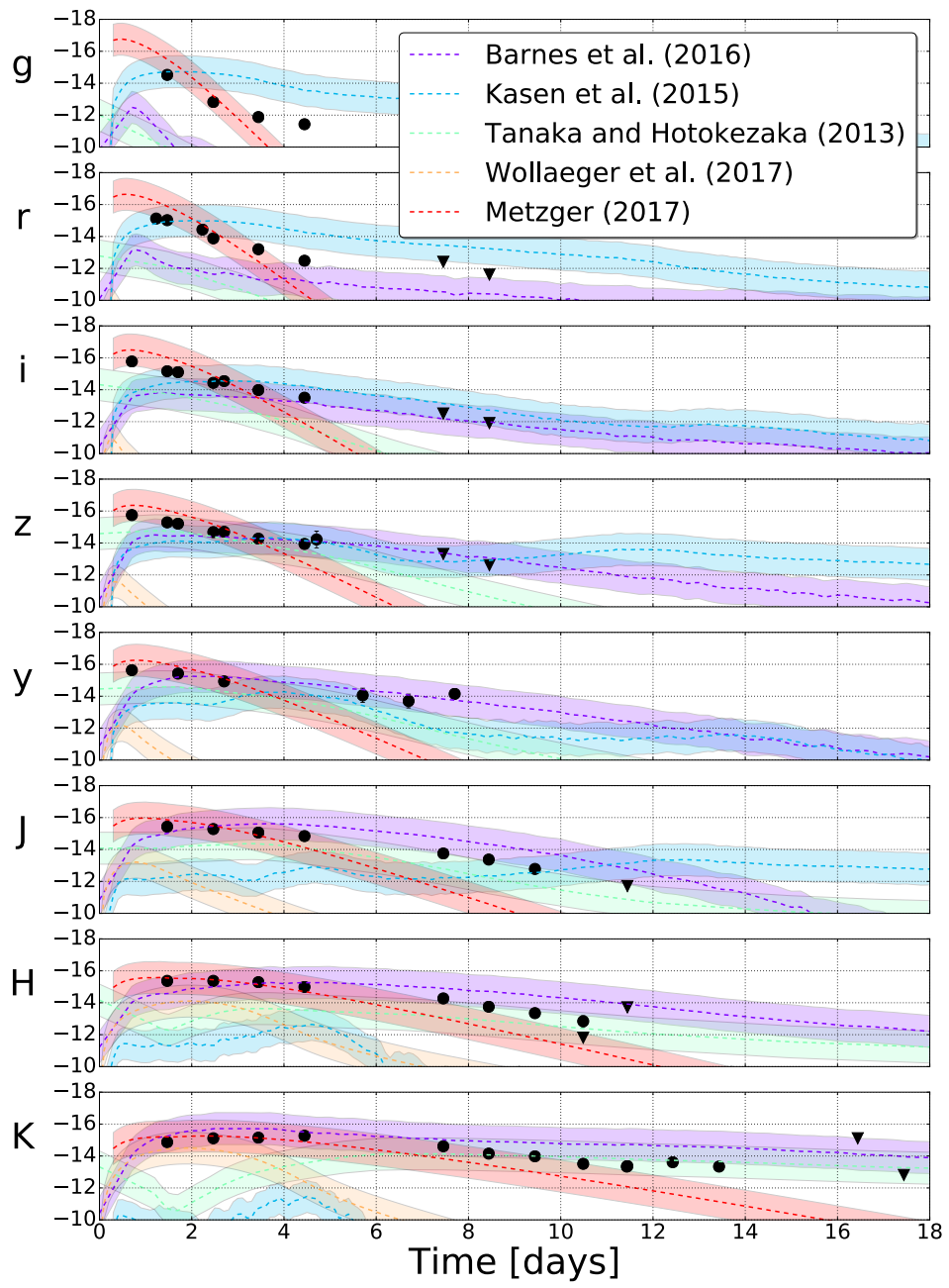

Figure 2: | Light curves of AT2017gfo. The combined photometry and five kilonova models $32[18,19]$ predicted before this discovery. From Barnes et al $\stackrel{18}{ }$, we use a model with $M_{\mathrm{ej}} \approx 5 \times 10^{-2} M_{\odot}$ and $v_{\mathrm{ej}} \approx$ $0.2 c$. From Kasen et al. $\frac{32}{\text {, we use the }} t 300$ disk wind outflow model corresponding to a simulation where the resultant neutron star survives $300 \mathrm{~ms}$ before collapsing to a black hole. From Tanaka \& Hotokezaka ${ }^{4}$, we use a model of a binary neutron star merger with masses 1.2 and $1.5 M_{\odot}$ assuming the APR4 equation of

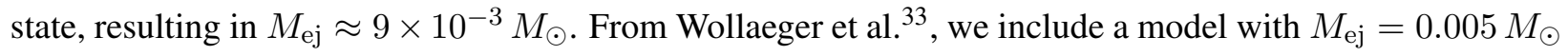
and $v_{\mathrm{ej}}=0.2 c$. From Metzge! $\frac{19}{19}$, we use a model with $M_{\mathrm{ej}} \approx 5 \times 10^{-2} M_{\odot}, v_{\mathrm{ej}} \approx 0.2 c, \alpha=3.0$, and $\kappa_{r}=0.1 \mathrm{~cm}^{2} \mathrm{~g}^{-1}$. 

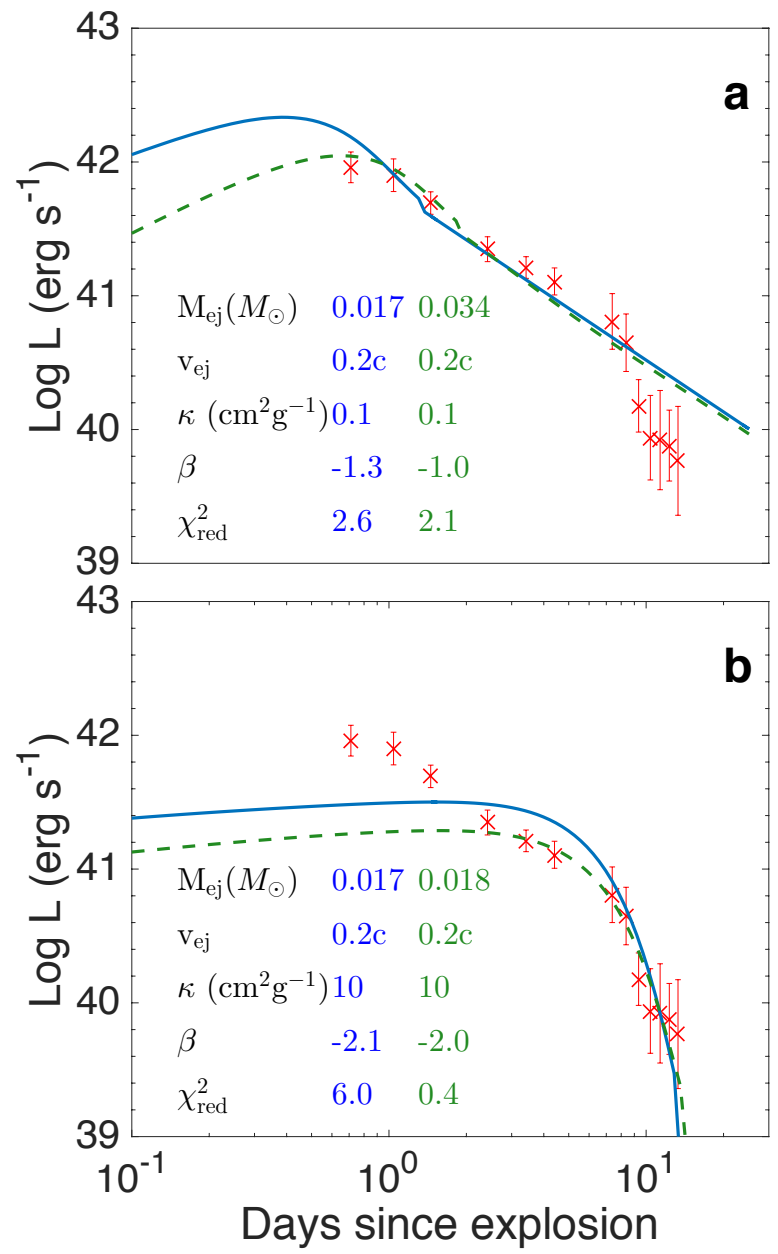

Figure 3: | Model light curve fits using the Arnett formalism. Mass, velocity, opacity $(\kappa)$ and a powerlaw slope for radioactive powering $(\beta)$ are freely variable. Each of these parameters were allowed to vary to give the best fit (reduced $\chi^{2}$ are quoted). a: The blue solid line shows the best fit. The green dashed model includes also a thermalization efficiency 19 . The recovered power law $(\beta=-1.0$ to -1.3$)$ is close to the one predicted in kilonova radioactivity models $(\beta=-1.2)$. b: Best fits when opacity is forced to $\kappa=10 \mathrm{~cm}^{2} \mathrm{~g}^{-1}$, to all data (blue, solid) and excluding first three data points (green, dashed). In all models the maximum allowed velocity is $0.2 \mathrm{c}$, which is also the preferred fit value. The errors are $1 \sigma$ uncertainties on the data, while the later points after 10 days are uncertain due to systematic effects. The full MCMC analysis and uncertainties are discussed in the Methods section. 

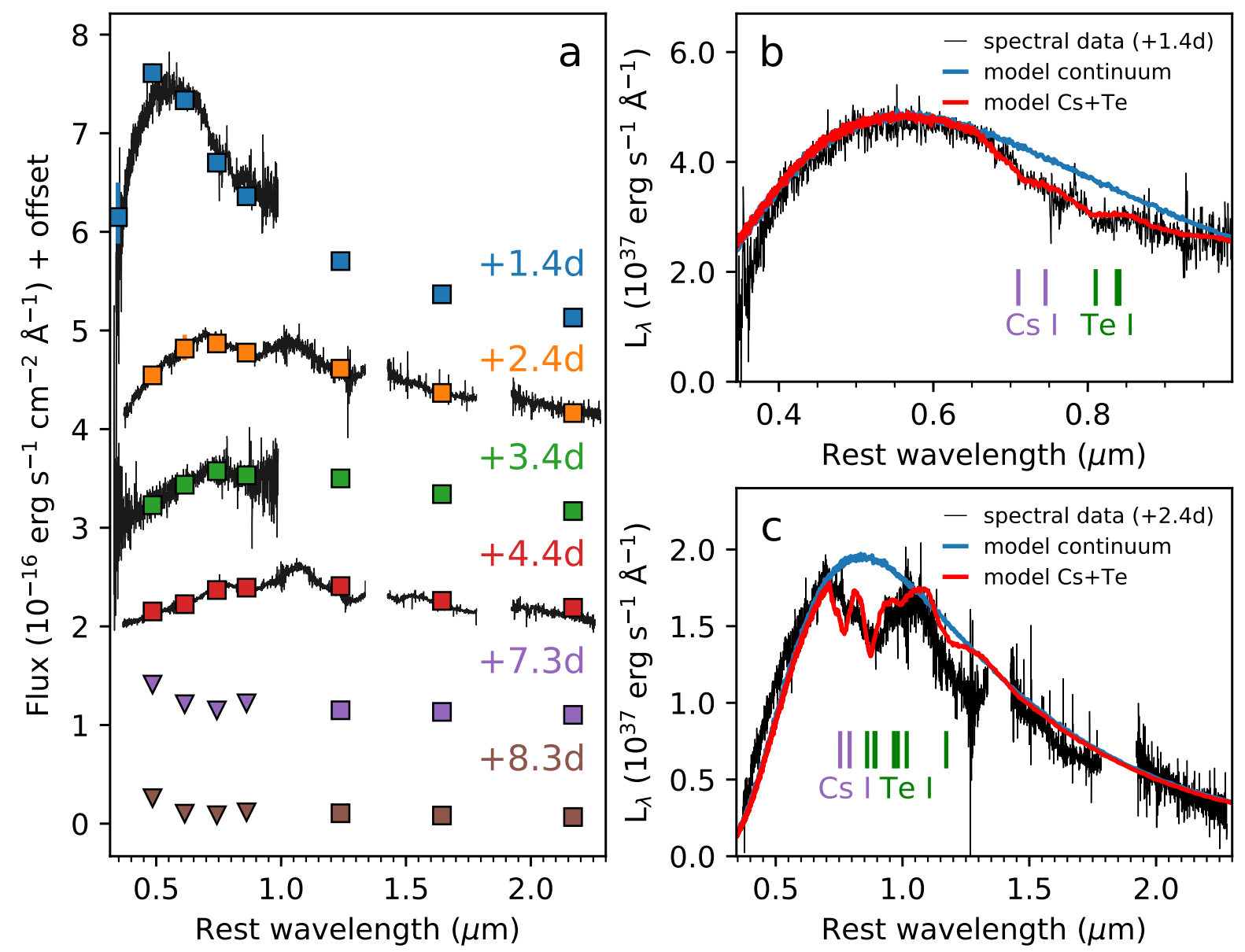

Figure 4: | Spectroscopic data and model fits a: Spectroscopic data from +1.4 to +4.4 days after discovery, showing the fast evolution of the SED. The points are coeval UgrizJHK photometry. b: Comparison of the +1.4 day spectrum with a TARDIS spectral model that includes Cs I and Te I [see text]. Thin vetical lines indicate the positions of spectral lines blueshifted by $0.2 \mathrm{c}$, corresponding to the photospheric velocity of the model (the adopted black-body continuum model is also shown for reference). c: The Xshooter spectrum at +2.4 days, also shows Cs I and Te I lines that are consistent with the broad features observed in the optical and near infra-red (here, the lines are indicated at velocities of $0.13 \mathrm{c}$ and we include additional, longer wavelength transitions to supplement those in B.). 


\section{Methods}

Distance and Reddening The host galaxy NGC4993 has been identified as a member of a group of 10 galaxies (LGG 332) ${ }^{34}$. The heliocentric recessional velocity of $2951 \pm 26 \mathrm{~km} \mathrm{~s}^{-1}$, or $z=0.009843 \pm 0.000087$, is from optical data ${ }^{35}$. The kinematic distance (correcting for various infall models and using $H_{0}=71 \pm 2 \mathrm{~km} \mathrm{~s}^{-1} \mathrm{Mpc}^{-1}$ ) and the Tully Fisher distances to the group containing NGC4993 $3^{36}$ are in good agreement within the uncertainty of $d=40 \pm 4 \mathrm{Mpc}$ (distance modulus $\mu=33.01 \pm 0.20$ ), and we adopt this value. The foreground reddening values in the direction of NGC4993 and AT2017gfo (as reported in NED' are adopted to be $A_{U}=$ 0.54, $A_{g}=0.39, A_{r}=0.28, A_{i}=0.21 A_{z}=0.16, A_{y}=0.13, A_{J}=0.09, A_{H}=0.06, A_{K}=0.04$ (Landolt $U$, Pan-STARRS1 grizy $y_{\mathrm{P} 1}$ and UKIRT $J H K$ ), or $E(B-V)=0.11$ mag. These reddening corrections were applied to the photometry to calculate absolute magnitudes and bolometric luminosities.

Hubble Space Telescope pre-discovery data NGC4993 was observed by the Hubble Space Telescope using the Advanced Camera for Surveys (ACS) Wide Field Channel on 2017 April 28, less than four months prior to the discovery of AT2017gfo. $2 \times 348 \mathrm{~s}$ exposures were taken with the F606W filter (comparable to Sloan $r^{\prime}$ ). As this is the deepest image of the site of AT2017gfo taken prior to discovery, we examined it for any possible pre-discovery counterpart.

We localised the position of AT2017gfo on the ACS image by aligning this to the GROND $i^{\prime}$ images taken on each of the nights from 2017 Aug 18 to 21 . Nine point sources common to

\footnotetext{
${ }^{1}$ The NASA/IPAC Extragalactic Database (NED) is operated by the Jet Propulsion Laboratory, California Institute of Technology, under contract with the National Aeronautics and Space Administration.
} 
both the GROND and ACS images were matched, and the final position on the ACS image has an uncertainty of 28, 50 mas in $x$ and $y$ respectively, determined from the scatter among the positions as measured on different GROND images.

No sources were detected by the DOLPHOT ${ }^{37}$ photometry package at a significance of $3 \sigma$ or higher, within a radius $>3 \times$ the positional uncertainty. We determined the limiting magnitude at the position of AT2017gfo to be F606W > 27.5 (VEGAMAG), based on the average magnitude of sources detected at $3 \sigma$ within a $100 \times 100$ pixel region centred on the position of AT2017gfo. For our adopted distance modulus and foreground reddening, this implies that any source at the position of AT2017gfo must have an absolute magnitude F606W $>-5.8$.

\section{ATLAS system and observational data and upper limit to the rate of kilonova events The} Asteroid Terrestrial-impact Last Alert System (ATLAS) ${ }^{38}$, is a full-time near Earth asteroid survey. It is currently running two $0.5 \mathrm{~m} \mathrm{f} / 2$ wide-field telescopes on Haleakala and Mauna Loa. The ATLAS sensor is a single thermoelectrically-cooled STA1600 detector with 1.86 arcsecond per pixel platescale $(10.56 \mathrm{k} \times 10.56 \mathrm{k}$ pixels $)$ giving a 29.2 square degree field of view. The two units work in tandem to survey the entire visible sky from $-40^{\circ}<\delta<80^{\circ}$ with a cadence of two to four days, depending on weather. The ATLAS unit on Haleakala has been working in scientific survey mode since April 2016 and was joined by the Mauna Loa unit in March 2017.

ATLAS observes in two wide-band filters, called "cyan" or " $c$ ", which roughly covers the SDSS/Pan-STARRS $g$ and $r$ filters, and "orange" or " $O$ ", which roughly covers the SDSS/PanSTARRS $r$ and $i$. The observing cadence for identifying moving asteroids is typically to observe 
each footprint 4-5 times (30 s exposures, slightly dithered) within about an hour of the first observation of each field. All data immediately go through an automatic data processing pipeline. This produces de-trended, sky-flattened images which are astrometrically corrected to the Gaia stellar reference frame and photometrically corrected using Pan-STARRS 1 reference stars ${ }^{16}$. Difference images are produced using a static-sky template and source extraction is carried out on both the target and difference images using DOPHOT on the target frames ${ }^{39}$ and a custom written package for PSF-fitting photometry, which we call TPHOT (on the difference frames). Sources found on the difference images are then cataloged in a MySQL database and merged into astrophysical objects if there are at least three detections from the five (or more) images. These objects are subject to a set of quality filters, a machine-learning algorithm and human scanning 16 40.

Our database did not contain any astrophysical object at the position of AT2017gfo between MJD 57380.64463 and 57966.26370. The position was observed 414 times and on each of these we forced flux measurements at the astrometric position of the transient on the difference image. We measured $5 \sigma$ flux limits and any epochs with greater than $5 \sigma$ detections. The $5 \sigma$ flux limits were in the range $o>18.6 \pm 0.5$ (AB mag, median and standard deviation) and $c>19.3 \pm 0.4$ (see Extended Data Figure 2). We found 44 images which formally had flux detections greater than $5 \sigma$, but on visual inspection we rule out these being real flux variability at the transient position. They all appear to be residuals from the host galaxy subtraction. With ATLAS, we rule out any variability down to 18.6 to 19.3 (filter dependent) during a period 601 to 16 days before discovery of AT2017gfo.

We can estimate an approximate upper limit to the rates of these kilonovae, without a GW 
trigger from the ATLAS survey. Extended Data Figure 2 implies that we would be sensitive to objects like AT2017gfo to $60 \mathrm{Mpc}$. ATLAS typically surveys 5000 sq deg per night, $4-5$ times, which provides a sampled volume of $10^{-4} \mathrm{Gpc}^{3}$ within $60 \mathrm{Mpc}$. If we assume that a kilonova lightcurve is visible for 4 days and we have observations every 2 - 4 days, and observe $60 \%$ of clear time then the control time is $0.9 \mathrm{yr}$. We have no candidates, therefore the simple Poisson probabilities of obtaining a null result are 50\%, 16\% and 5\% when the expected values are 0.7 , 1.8 and $3.0 \times 10^{4} \mathrm{Gpc}^{-3} \mathrm{yr}^{-1}$. Therefore the $95 \%$ confidence upper limit to the rate of kilonovae is $<3.0 \times 10^{4} \mathrm{Gpc}^{-3} \mathrm{yr}^{-1}$. This simple approach is in broad agreement with the upper limit from the Dark Energy Survey ${ }^{41}$ and the LIGO Scientific collaboration for NS-NS mergers ${ }^{42}$ and a more sophisticated calculation is warranted for the ATLAS data.

The Pan-STARRS1 system and observational data The Pan-STARRS1 system ${ }^{43}$ comprises a $1.8 \mathrm{~m}$ telescope with a 1.4 Gigapixel camera (called GPC1) mounted at the Cassegrain $f / 4.4$ focus. This wide-field system is located on the summit of Haleakala on the Hawaiian island of Maui. The GPC1 is composed of sixty Orthogonal Transfer Array devices (OTAs), each of which has a detector area of $48460 \times 48680$ pixels. The pixels are 10 microns in size $(0.26$ arcsec $)$ giving a focal plane of $418.88 \mathrm{~mm}$ in diameter or 3.0 degrees. This corresponds to field-of-view area of 7.06 square degrees, and an active region of about 5 square degrees. The filter system (which we denote grizy $_{\mathrm{P} 1}$ ) is similar to the $\operatorname{SDSS}^{44}$ and is described in detail in two papers ${ }^{43}[45$. Images from Pan-STARRS1 are processed immediately with the Image Processing Pipeline ${ }^{46}$. The existence of the Pan-STARRS1 $3 \pi$ Survey dat ${ }^{43}$ provides a ready made template image of the whole sky north of $\delta=-30^{\circ}$, and we furthermore have proprietary $i_{\mathrm{P} 1}$ data in a band between $-40^{\circ}<\delta<-30^{\circ}$, 
giving a reference sky in the $i_{\mathrm{P} 1}$ band down to this lower declination limit. Images in $i_{\mathrm{P} 1} z_{\mathrm{P} 1} y_{\mathrm{P} 1}$ were taken on 7 nights, at high airmass due to the position of AT2017gfo.

A series of dithered exposures were taken in the three filters during the first available night (starting 2017 Aug 18 05:33:01 UT), and we placed the target on a clean detector cell. We repeated the $i_{\mathrm{P} 1} z_{\mathrm{P} 1} y_{\mathrm{P} 1}$ for two subsequent nights until the object became too low in twilight and we switched to $z_{\mathrm{P} 1}$ and $y_{\mathrm{P} 1}$ and then only $y_{\mathrm{P} 1}$. Frames were astrometrically and photometrically calibrated with standard Image Processing Pipeline steps ${ }^{46}[47[48$. The Pan-STARRS1 $3 \pi$ reference sky images were subtracted from these frames ${ }^{49}$ and photometry carried out on the resulting difference $\operatorname{image}^{47}$.

ePESSTO and Xshooter observational data EFOSC 2 consists of a combined $2048 \times 2048$ pixel CCD imaging camera and low-dispersion spectrograph, mounted at the Nasmyth focus of the $3.58 \mathrm{~m}$ New Technology Telescope (NTT) at La Silla, Chile. The SOFI instrument has a $1024 \times 1024$ pixel near infra-red array for long-slit spectroscopy and imaging, and is also mounted at the NTT on the other Nasmyth focus. All EFOSC2 spectra were taken at the parallactic angle using the configurations listed in Table 1 , and reduced using the PESSTO pipeline ${ }^{15}$. Spectroscopic frames were trimmed, overscan and bias subtracted, and divided by a normalised flat field. In the case of the Gr\#16 spectra, a flat field was obtained immediately after each spectrum to enable fringing in the red to be corrected. Spectra were wavelength calibrated using arc lamps, and the wavelength solution checked against strong sky emission lines. Cosmic rays were masked in the two-dimensional spectra using the LACosmic algorithm ${ }^{50}$, before one-dimensional spectra were optimally extracted from each frame. Flux calibration of the spectra was done using an average sensitivity curve de- 
rived from observations of several spectrophotometric standard stars during each night, while the telluric features visible in the red were corrected using a synthetic model of the absorption.

The Xshooter instrument on the ESO Very Large Telescope was used for two epochs of spectra. The observational setup and spectral reductions were similar to those previously employed in and detailed in several publications ${ }^{51} \sqrt[52]{5}$, with the custom-built T. Krühler reduction pipeline used for the reduction and flux calibration and molecfit package used for telluric correction. All spectra were scaled to contemporaneous photometric flux calibrations. Images with the NACO and VISIR instruments on the ESO Very Large Telescope were taken in the $L$-band (NACO) and $N$-band (VISIR) in the mid-infrared. These were kindly made public by ESO to all collaborating groups working with the LIGO-Virgo follow-up programmes and are publicly available through the ESO archive. We found no detection of the transient in either instrument. The host galaxy NGC4993 was faint, but visible in the $L$-band NACO images. With only one standard star, at a vastly different airmass from the target we could not reliably determine an upper limit. Similarly, no flux was visible in the VISIR $N$-band data.

The EFOSC2 and SOFI images were reduced using the PESSTO pipeline. All EFOSC2 images were overscan and bias subtracted, and divided by a flat-field frame created from images of the twilight sky. Individual images taken at each epoch were then aligned and stacked. The SOFI images were cross-talk and flat-field corrected, sky subtracted, aligned, and merged. The transient had faded below the detection limit in the $g^{\prime} r^{\prime} i^{\prime} z^{\prime}$ GROND images obtained on 2017 August 26.97 UT, and the $U$ EFOSC2 image observed on 2017 August 21.05 UT. The VISTA Hemisphere survey $J K_{\mathrm{s}}$ images observed on 2014 April 10 were used as references for the SOFI $J K_{\mathrm{s}}$ images. No 
VISTA archive images were available in $H$-band, therefore we used the GROND $H$-band on 2017 Aug 29.99 UT as the reference. Template image subtraction to remove the contribution from the host galaxy was carried out based on the ISIS2.2 package ${ }^{53}$, and the subtractions were of good quality. Point-spread function (PSF) fitting photometry was carried out on each stacked and template-subtracted image. An empirical model of the PSF was made for each image from sources in the field, and fitted to the transient to determine its instrumental magnitude. In the case where the transient was not detected, artificial star tests were used to set a limiting magnitude. The photometric zeropoint for each image was determined through aperture photometry of PanSTARRS1 or 2MASS sources in the field of the EFOSC2 and SOFI images, respectively, and used to calibrate the instrumental magnitudes onto a standard system. Three further epochs were taken with the Boyden 1.52-m telescope in South Africa, giving extra time resolution coverage over the first $72 \mathrm{hrs}$. The Boyden 1.52-m telescope, is a $1.52 \mathrm{~m}$ Cassegrain reflector combined with an Apogee $1152 \times 770$ pixel CCD imaging camera, providing a field of view of 3.7 arcmin $\times$ 2.5 arcmin. Observations were carried out during twilight and the early hours of the night at low altitude using $30 \mathrm{sec}$ exposures. Observations were reduced and analysed using a custom pipeline for this telescope. All photometric observations were taken using a clear filter and then converted to SDSS r using four Pan-STARRS1 reference stars.

GROND system and observational data Observations with GROND ${ }^{54}$ at the $2.2 \mathrm{~m}$ Max-Planck telescope at La Silla ESO started on 2017 Aug 18 23:15 UT $T^{[55}$. Simultaneous imaging in $g^{\prime} r^{\prime} i^{\prime} z^{\prime} J H K_{\mathrm{s}}$ continued daily, weather allowing until 2017 Sept 4 (see Tables 2 and 3). GROND data were reduced in the standard manner using pyraf/IRAF $\stackrel{56}{ }$. PSF photometry of field stars was calibrated 
against catalogued magnitudes from Pan-STARRS 1 $1^{43}$ for $g^{\prime} r^{\prime} i^{\prime} z^{\prime}$ images and 2MASS for $J H K_{\mathrm{s}}$ images. The images were template subtracted using the ISIS2.2 package $e^{53}$. GROND $g^{\prime} r^{\prime} i^{\prime} z^{\prime}$ images from 2017 Aug 26.97 UT and $J H K_{\mathrm{s}}$ images from 2017 Aug 29.99 UT were used as reference images. These were the best quality images we had with no detection of the source. The photometry results in typical absolute accuracies of \pm 0.03 mag in $g^{\prime} r^{\prime} i^{\prime} z^{\prime}$ and \pm 0.05 mag in $J H K_{\mathrm{s}}$.

Spectral and lightcurve comparisons A comparison of our spectra with a sample of $\mathrm{SNe}$ is shown in Extended Data Fig. 3. Both the spectral shape and features present differ significantly, with AT2017gfo showing a significantly redder SED than those of either Type Ia or Type II-P SNe within a few days of explosion. The spectra of AT2017gfo also lack the typical absorption features of intermediate-mass elements that are normally seen in early-time SN spectra. Fig. 3 also shows a comparison with optical spectra from a sample of some of the faintest and fastest evolving Type I SN discovered to date.

PESSTO has spectroscopically classified 1160 transients, and monitored 264, and none are similar to AT2017gfo. Volume-limited samples of supernovae (having samples of around 100-200 SNe within 30-60 Mpc) have never uncovered a similar transient 57 58. In the ATLAS survey, during the period up to Aug 2017, we have found 75 transients (all supernovae) in galaxies within $60 \mathrm{Mpc}$ and no objects like AT2017gfo. This implies that objects like AT2017gfo have a rate of around 1\% or less of the local supernova rate, justifying our probability calculation in the main text.

Bolometric light curve calculation Firstly, the broad-band magnitudes in the available bands $(U$, $g, r, i, z, y, J, H, K_{s}$ ) were converted into fluxes at the effective filter wavelengths, and then 
corrected for the adopted extinctions (see Methods - Distance and reddening). For completeness at early phases, we ensured consistency with the values for ultra-violet flux reported from the Swift public data in the bands $u v w 2, u v m 2$, uvw1, and $U^{5960}$. An SED was then computed over the wavelengths covered. Fluxes were converted to luminosities using the distance previously adopted. We determined the points on the bolometric light curve at epochs when $K$-band or ultraviolet observations were available. Magnitudes from the missing bands were generally estimated by interpolating the light curves using low-order polynomials $(n \leq 2)$ between the nearest points in time. We also checked that the interpolated/extrapolated magnitudes were consistent with the available limits. Finally, we fitted the available SED with a black-body function and integrated the flux from $1000 \AA$ to $25000 \AA$. This provides a reasonable approximation to the full bolometric light curve ${ }^{\sqrt{61}}$ but we caution that flux beyond $25000 \AA$ may contribute. It is not clear that the spectral energy distribution at this phase is physically well represented by a black body, and therefore we chose not to integrate fully under such a spectrum. Therefore the bolometric flux that we estimate at 8 days and beyond could be higher. For reference we report the temperature and radius evolution, together with uncertainties, from the SED fitting in Table 4, although we again note that a black body assumption may not be valid at later times.

Light curve modelling - parameter range estimation We compare the light curve data with the models by Arnett and Metzger using a Bayesian framework ${ }^{62}$. The likelihood in our case is defined as $\mathcal{L}=e^{-\chi^{2} / 2}$. The time of the kilonova (used on both models) is defined to be that of the gravitational-wave trigger time. For both the Metzger and Arnett models considered in this analysis, we choose a $\log$ uniform prior of $-5 \leq \log _{10}\left(M_{\mathrm{ej}}\right) \leq 0$ for the ejecta mass, a uniform 
prior of $0 \leq v_{\mathrm{ej}} \leq 0.3 \mathrm{c}$ for the ejecta velocity, and a uniform prior of $-1 \leq \log _{10}(\kappa) \leq 2 \mathrm{~cm}^{2} \mathrm{~g}^{-1}$ for the opacity. Specifically for the Metzger model, we choose a uniform prior of $0 \leq \alpha \leq 10$ for the slope of the ejecta velocity distribution. The power-law slope for radioactive powering given in the Arnett model is given a prior of $-5 \leq \beta \leq 5$.

We sample this given posterior using a nested sampling approach using the MULTINEST implementation ${ }^{63}$ through a PYTHON wrapper ${ }^{64}$. Figure 4 shows the posterior of the Arnett model. Figure 5 shows the posterior of the Metzger model.

Systematic error for mass is dominated by uncertainty in the heating rate per mass of the ejecta. This consists of the product of intrinsic decay power, and thermalization efficiency. For the intrinsic decay power, we find values of $(1-3) \times 10^{10} \mathrm{erg} \mathrm{g}^{-1} \mathrm{~s}^{-1}$ in the literature 2[24 [25] [19, $1.9 \times 10^{10} \mathrm{erg} \mathrm{g}^{-1} \mathrm{~s}^{-1}$ is our default value. There are only small uncertainties associated with nuclear mass models during the first few days, but this grows to a factor of $\sim 2-3$ at later times ${ }^{19}$.

Due to the dominance of the post-diffusion tail in the fits, the mass scales roughly inversely with the powering level. Thus, if this is a factor of two higher than assumed our mass range declines by a factor of 2 . However, the vast majority of decay models are close to our value, so we favour the $\sim 0.04 M_{\odot}$ solutions over the $\sim 0.02 M_{\odot}$ ones. We note also that even the high-opacity models fitting the later data points have $M \gtrsim 0.02 M_{\odot}$, so this should be a robust lower limit to the ejecta mass.

TARDIS Modelling details For the temperature implied by the black-body like SED, Fe would be expected to be primarily in its neutral or singly-ionized state: in either case, detectable features 
would be expected. In particular, the lack of evidence for Fe II features (e.g. the Fe II $\lambda 5064$ multiplet) in the blue part of our spectrum places a strong limit on the presence of this ion (simple TARDIS modelling suggests $<10^{-3} M_{\odot}$ of Fe II can be present in the spectral forming region). This lack of Fe partly argues against ejecta compositions dominated by Fe-peak elements. Equivalent constraints on $\mathrm{Ni}$, however, are weaker.

As noted in the main text, the combination of limited atomic data and simplistic modelling means that we cannot derive reliable elemental masses from the analysis carried out so far. However, we note that our model for the $+1.4 \mathrm{~d}$ spectrum invokes ion masses of only $\sim 10^{-9} \mathrm{M}_{\odot}$ and a few times $10^{-3} \mathrm{M}_{\odot}$ for Cs I and Te I, respectively, at ejecta velocities above the adopted photosphere (i.e. $v>0.2 \mathrm{c}$ ). In both cases, these are only lower limits on elemental masses, since the ions in question are expected to be sub-dominant at the conditions present in the ejecta (this is a particularly important consideration for Cs I, owing to its low ionization potential of only $3.9 \mathrm{eV}$ ). Nethertheless, these mass limits are consistent with the ejecta masses suggested in our light curve model.

Kilonova simulations Kilonova simulations predict two distinct ejecta components: dynamic ejecta and disk winds. The dynamic ejecta is expelled directly in the merger. Starting from neutron star material with $Y_{e} \sim 0.03$, it experiences some moderated de-neutronization by positron captures, but likely ends with $Y_{e} \lesssim 0.2$ (as described in simulations ${ }^{5}$ ). Such composition is predicted to produce all heavy r-process elements, including lanthanides and actinides. It is thus expected to lead to a high-opacity red component peaking on time scales of days/weeks. The disk wind has two components, a radiation driven wind and a dynamic torus ejection. These are exposed to neu- 
trino irradiation, which can produce a larger variation in $Y_{e}$. This component can thus be largely lanthanide and actinide free, and have low opacity, in particular for $0.2<Y_{e}<0.4$. Dynamic and wind ejecta have similar heating rates ${ }^{2}$. Thus, their contribution to the bolometric light curve is largely proportional to their masses. The compilation by Wu et al ${ }^{65}$ shows that current simulations predict similar masses of the two components, but uncertainty of a factor few for their mass ratio.

The data suggest that we have detected the lower-opacity disk wind component, and that this has a $Y_{e}$ in the range giving low opacity (giving constraints on the poorly understood $Y_{e}$ setting processes). Whether a dynamic component is present as well is harder to ascertain. The whole light curve is reasonably well fit by a single disk wind component. Our models are too simplistic to warrant exploration of two-component scenarios. Assuming we have detected a disk wind of several times $0.01 M_{\odot}$, it is not easy to make this component drop away enough at late times to leave much flux for a dynamic ejecta component. Perhaps the opacity in the dynamic ejecta is as high $\left(\kappa \gtrsim 100 \mathrm{~cm}^{2} \mathrm{~g}^{-1}\right)$ as speculated ${ }^{3] 66}$, and it then remains too dim to be seen compared to the wind for at least the first 20 days. Alternatively, this kilonova may simply have $M_{\text {wind }} \gg M_{\text {ejecta }}$. The only circumstance which could substantially change these conclusions is if the first $2-3$ data points are caused by a GRB afterglow. Then, a dynamic component with $\kappa \gtrsim 10 \mathrm{~cm}^{2} \mathrm{~g}^{-1}$, can reasonably well fit the later data points. However, as we discuss in the main paper we find several arguments against this scenario, such as the chromatic light curve evolution and the absence of a strong X-ray afterglow, and assess that the early light is caused by a blue kilonova.

Data Availability Statement The reduced, calibrated spectral data presented in this paper are openly 
available on the Weizmann Interactive Supernova data REPository (https://wiserep.weizmann.ac.il) and at the ePESSTO project website http://www.pessto.org. The raw data from the VLT, NTT and GROND (for spectra and imaging) are available from the ESO Science Archive facility http://archive.eso.org. The raw pixel data from Pan-STARRS1 and the 1.5m Boyden telescope are available from the authors on request.

Code Availability The lightcurve fitting code described here is publicly available at the following website: https://star.pst.qub.ac.uk/wiki/doku.php/users/ajerkstrand/start. A code to produce the posteriors in this paper is available at: https://github.com/mcoughlin/gwemlightcurves. TARDIS is an open-source Monte Carlo radiative-transfer spectral synthesis code for 1D models of supernova ejecta and is publicly available here https://tardis.readthedocs.io/en/latest/. Standard software within the IRAF environment was used to carry out the spectral, and imaging reductions and photometry.

Acknowlegdements This work is based on observations collected at the European Organisation for Astronomical Research in the Southern Hemisphere, Chile as part of ePESSTO, (the extended Public ESO Spectroscopic Survey for Transient Objects Survey) ESO program 199.D-0143 and 099.D-0376. We thank ESO staff for their excellent support at La Silla and Paranal and for making the NACO and VISIR data public to LIGO-Virgo collaborating scientists. We thank Jacob Ward for permitting a time switch on the NTT. PS1 and ATLAS are supported by NASA Grants NNX08AR22G, NNX12AR65G, NNX14AM74G and NNX12AR55G. Part of the funding for GROND was generously granted from the Leibniz-Prize to Prof. G. Hasinger (DFG grant HA 1850/28-1). Pan-STARRS1 and ATLAS are supported by NASA Grants NNX08AR22G, NNX12AR65G, NNX14AM74G and NNX12AR55G issued through the SSO Near Earth Object Observations Program. We acknowledge the excellent help in obtaining GROND data from Angela Hempel, Markus Rabus and Régis Lachaume on La Silla. The Pan-STARRS1 Surveys (PS1) were made possible by the IfA, University of Hawaii, the Pan-STARRS Project Office, the Max-Planck Soci- 
ety, MPIA Heidelberg and MPE Garching, Johns Hopkins University, Durham University, University of Edinburgh, Queen's University Belfast, Harvard-Smithsonian Center for Astrophysics, Las Cumbres Observatory Global Telescope Network Incorporated, National Central University of Taiwan, Space Telescope Science Institute, National Science Foundation under Grant No. AST-1238877, University of Maryland, and Eotvos Lorand University (ELTE) and the Los Alamos National Laboratory. We acknowledge EU/FP7-ERC Grants [291222], [615929] and STFC funding through grant ST/P000312/1 and ERF ST/M005348/1. AJ acknowledges Marie Sklodowska-Curie grant No 702538. MG, AH, KAR and ŁW thank Polish NCN grant OPUS 2015/17/B/ST9/03167, JS is funded by Knut and Alice Wallenberg Foundation. CB, MDV., NE-R., AP and GT are supported by the PRIN-INAF 2014. MC is supported by the David and Ellen Lee Prize Postdoctoral Fellowship at the California Institute of Technology. MF is supported by a Royal Society - Science Foundation Ireland University Research Fellowship. MS and CI acknowledge support from EU/FP7-ERC grant no [615929]. PGJ acknowledges the ERC consolidator grant number [647208]. GREAT is funded by VR. JDL gratefully acknowledges STFC grant ST/P000495/1. TWC, PS and PW acknowledge the support through the Alexander von Humboldt Sofja Kovalevskaja Award. JH acknowledges financial support from the Vilho, Yrjö and Kalle Väisälä Foundation. JV acknowledges FONDECYT grant number 3160504. LG was supported in part by the US National Science Foundation under Grant AST-1311862. MB acknowledges support from the Swedish Research Council and the Swedish Space Board. AG-Y is supported by the EU via ERC grant No. 725161, the Quantum Universe I-Core program, the ISF, BSF and by a Kimmel award. LS acknowledges IRC grant GOIPG/2017/1525. AJR is supported by the Australian Research Council Centre of Excellence for All-sky Astrophysics (CAASTRO) through project number CE110001020. IRS was supported by the Australian Research Council Grant FT160100028. We acknowledge Millennium Science Initiative grant IC120009. This paper uses observations obtained at the Boyden Observatory, University of the Free State, South Africa. 
34. Makarov, D. \& Karachentsev, I. Galaxy groups and clouds in the local (z 0.01) Universe. Mon. Not. R. Astron. Soc. 412, 2498-2520 (2011). 1011.6277.

35. Wegner, G. et al. Redshift-Distance Survey of Early-Type Galaxies: Spectroscopic Data. Astron. J. 126, 2268-2280 (2003). astro-ph/0308357.

36. Freedman, W. L. et al. Final Results from the Hubble Space Telescope Key Project to Measure the Hubble Constant. Astrophys. J. 553, 47-72 (2001). astro-ph/ 0012376.

37. Dolphin, A. DOLPHOT: Stellar photometry. Astrophysics Source Code Library (2016). 1608.013

38. Tonry, J. L. An Early Warning System for Asteroid Impact. Publications of the Astronomical Society of the Pacific 123, 58-73 (2011). 1011.1028.

39. Schechter, P. L., Mateo, M. \& Saha, A. DOPHOT, a CCD photometry program: Description and tests. Publications of the Astronomical Society of the Pacific 105, 1342-1353 (1993).

40. Smartt, S. J. et al. Pan-STARRS and PESSTO search for an optical counterpart to the LIGO gravitational-wave source GW150914. Mon. Not. R. Astron. Soc. 462, 4094-4116 (2016). 1602.04156.

41. Doctor, Z. et al. A Search for Kilonovae in the Dark Energy Survey. Astrophys. J. 837, 57 (2017). 1611.08052. 
42. Abbott, B. P. et al. Upper Limits on the Rates of Binary Neutron Star and Neutron Star-Black Hole Mergers from Advanced LIGO's First Observing Run. Astrophys. J. Letters 832, L21 (2016). 1607.07456.

43. Chambers, K. C. et al. The Pan-STARRS1 Surveys. ArXiv e-prints (2016). 1612.05560.

44. Abazajian, K. N. et al. The Seventh Data Release of the Sloan Digital Sky Survey. Astrophys. J. Supplements 182, 543-558 (2009). 0812.0649.

45. Tonry, J. L. et al. The Pan-STARRS1 Photometric System. Astrophys. J. 750, 99 (2012). 1203.0297

46. Magnier, E. A. et al. Pan-STARRS Data Processing System. ArXiv e-prints (2016). 1612 . 05240.

47. Magnier, E. A. et al. Pan-STARRS Pixel Analysis : Source Detection \& Characterization. ArXiv e-prints (2016). 1612.05244.

48. Magnier, E. A. et al. Pan-STARRS Photometric and Astrometric Calibration. ArXiv e-prints (2016). 1612.05242.

49. Waters, C. Z. et al. Pan-Starrs Pixel Processing: Detrending, Warping, Stacking. ArXiv eprints (2016). 1612.05245

50. van Dokkum, P. G. Cosmic-Ray Rejection by Laplacian Edge Detection. Publications of the Astronomical Society of the Pacific 113, 1420-1427 (2001). astro-ph/0108003. 
51. Krühler, T. et al. GRB hosts through cosmic time. VLT/X-Shooter emission-line spectroscopy of $96 \gamma$-ray-burst-selected galaxies at $0.1<z<3.6$. Astron. Astrophys. 581, A125 (2015).

\subsection{3.}

52. Jerkstrand, A. et al. Long-duration Superluminous Supernovae at Late Times. Astrophys. J. 835, 13 (2017). 1608.02994.

53. Alard, C. \& Lupton, R. H. A Method for Optimal Image Subtraction. Astrophys. J. 503, 325-331 (1998). astro-ph/9712287.

54. Greiner, J. et al. GROND a 7-Channel Imager. Publications of the Astronomical Society of the Pacific 120, 405 (2008). 0801.4801.

55. Wiseman, P., Chen, T.-W., Greiner, J. \& Schady, P. 21584. GRB Coordinates Network 21572 (2017).

56. Krühler, T. et al. The $2175 \AA$ A Dust Feature in a Gamma-Ray Burst Afterglow at Redshift 2.45. Astrophys. J. 685, 376-383 (2008). 0805.2824.

57. Li, W. et al. Nearby supernova rates from the Lick Observatory Supernova Search - II. The observed luminosity functions and fractions of supernovae in a complete sample. Mon. Not. R. Astron. Soc. 412, 1441-1472 (2011). 1006.4612 .

58. Eldridge, J. J., Fraser, M., Smartt, S. J., Maund, J. R. \& Crockett, R. M. The death of massive stars - II. Observational constraints on the progenitors of Type Ibc supernovae. Mon. Not. R. Astron. Soc. 436, 774-795 (2013). 1301.1975. 
59. Evans, P., Kennea, J., Breeveld, A., Campana, S. \& et al. 21550. GRB Coordinates Network 21550 (2017).

60. Cenko, S., Emery, S., Campana, S., Evans, P. \& et al. 21572. GRB Coordinates Network 21572 (2017).

61. Inserra, C. et al. Complexity in the light curves and spectra of slow-evolving superluminous supernovae. Mon. Not. R. Astron. Soc. 468, 4642-4662 (2017). 1701.00941.

62. Coughlin, M. et al. Towards rapid transient identification and characterization of kilonovae. ArXiv e-prints (2017). 1708.07714.

63. Feroz, F., Hobson, M. P. \& Bridges, M. MultiNest: an efficient and robust Bayesian inference tool for cosmology and particle physics. Mon. Not. Roy. Astron. Soc. 398, 1601-1614 (2009). 0809.3437

64. Buchner, J., Georgakakis, A., Nandra, K. et al. X-ray spectral modelling of the AGN obscuring region in the CDFS: Bayesian model selection and catalogue. Astron. Astrophys. 564, A125 (2014). 1402.0004

65. Wu, M.-R., Fernández, R., Martínez-Pinedo, G. \& Metzger, B. D. Production of the entire range of r-process nuclides by black hole accretion disc outflows from neutron star mergers. Mon. Not. R. Astron. Soc. 463, 2323-2334 (2016). 1607.05290.

66. Tanaka, M. et al. Properties of Kilonovae from Dynamical and Post-Merger Ejecta of Neutron Star Mergers. ArXiv e-prints (2017). 1708.09101. 
67. Perets, H. B. et al. A faint type of supernova from a white dwarf with a helium-rich companion. Nature 465, 322-325 (2010). 0906.2003.

68. Drout, M. R. et al. The Fast and Furious Decay of the Peculiar Type Ic Supernova 2005ek. Astrophys. J. 774, 58 (2013). 1306.2337.

69. Kasliwal, M. M. et al. Rapidly Decaying Supernova 2010X: A Candidate ".Ia" Explosion. Astrophys. J. Letters 723, L98-L102 (2010). 1009.0960.

70. Valenti, S. et al. PESSTO monitoring of SN 2012hn: further heterogeneity among faint Type I supernovae. Mon. Not. R. Astron. Soc. 437, 1519-1533 (2014). 1302.2983.

71. Inserra, C. et al. OGLE-2013-SN-079: A Lonely Supernova Consistent with a Helium Shell Detonation. Astrophys. J. Letters 799, L2 (2015). 1410.6473.

72. Mazzali, P. A. et al. Hubble Space Telescope spectra of the Type Ia supernova SN 2011fe: a tail of low-density, high-velocity material. Mon. Not. R. Astron. Soc. 439, 1959-1979 (2014). 1305.2356

73. Hamuy, M. et al. The Distance to SN 1999em from the Expanding Photosphere Method. Astrophys. J. 558, 615-642 (2001). 
Extended Data
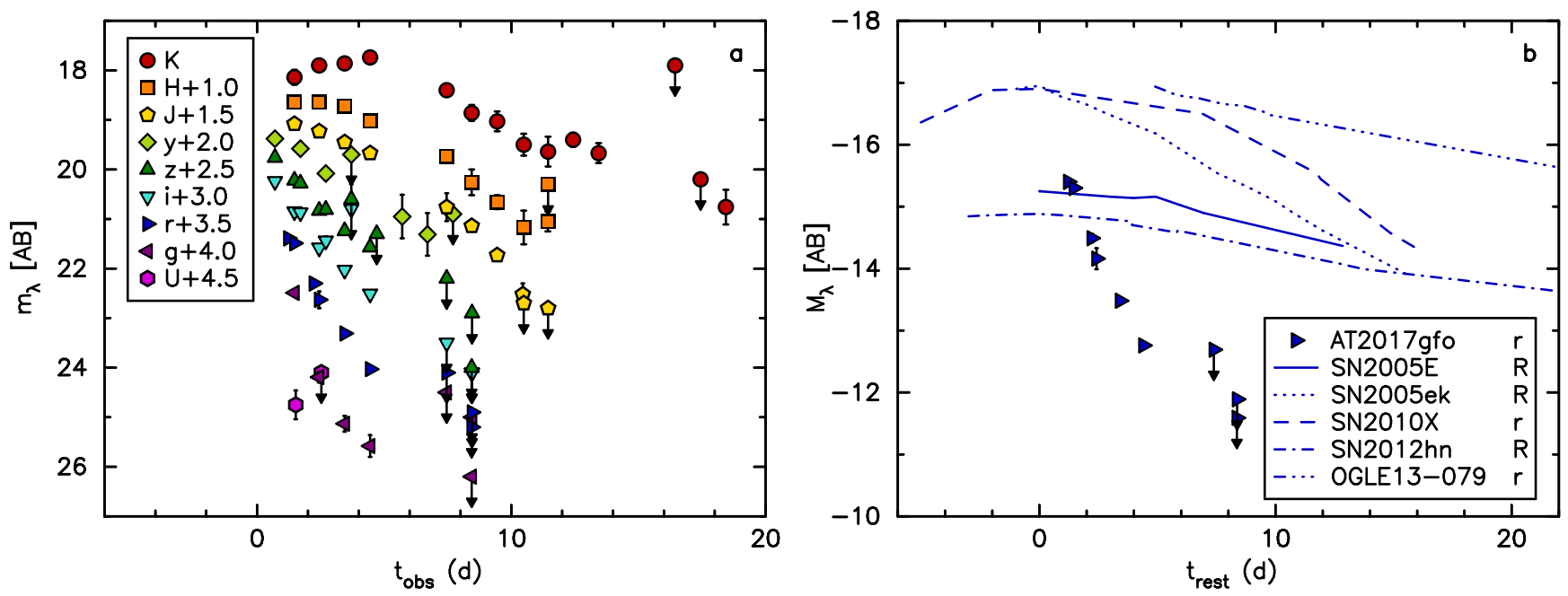

Extended Data Figure 1 | Light curves of AT2017gfo a) Observed AB light curves of AT2017gfo, vertically shifted for clarity. The $1 \sigma$ uncertainties are typically smaller than the symbols. b) Comparison of the absolute $r$-band light curve of AT2017gfo with those of a selection of faint

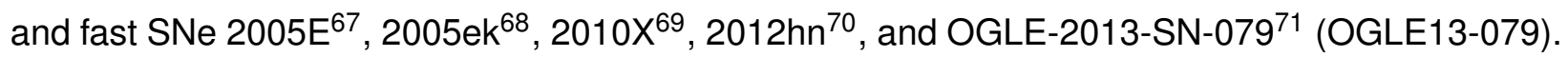
The comparison event phases are with respect to maximum light, while for AT2017gfo with respect to the LIGO trigger. 


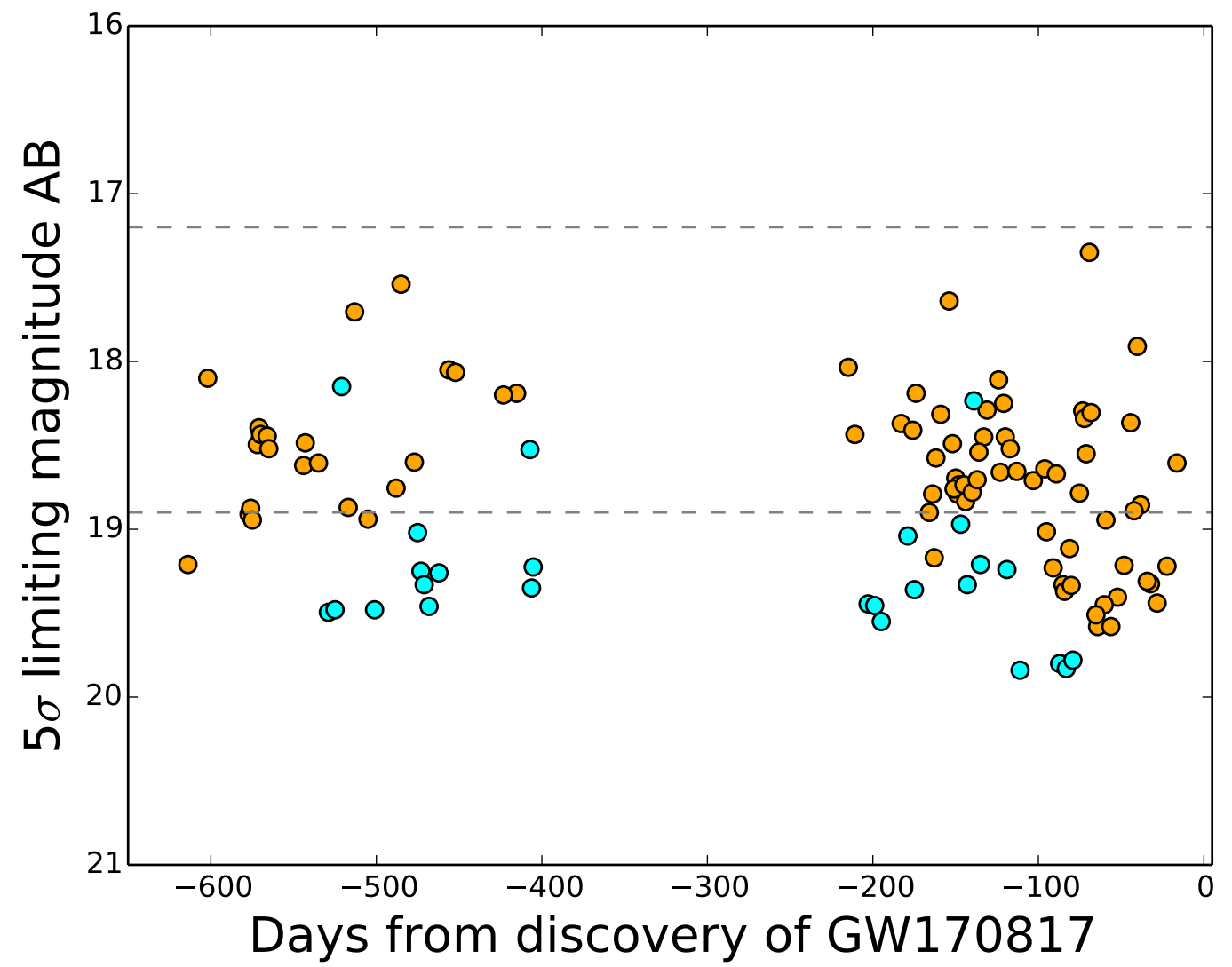

Extended Data Figure 2 | ATLAS limits at the position of AT2017gfo: $5 \sigma$ upper limits (from forced photometry) at the position of AT2017gfo up to 601 days before discovery in the ATLAS images. The cyan and orange filter limits are plotted as those colours. These limits are measured on the difference images, which are the individual $30 \mathrm{sec}$ frames after having the ATLAS reference sky subtracted off. The points plotted represent (typically) 5 images per night, and are the median limits of those five $30 \mathrm{sec}$ frames. The two horizontal lines indicate the $A B$ orange mag of AT2017gfo at 0.7 and 2.4 days after discovery, illustrating that ATLAS has sensitivity to make discoveries within 1-2 days of NS-NS merger at this distance. The last non-detection is 16 days before discovery of AT2017gfo. 

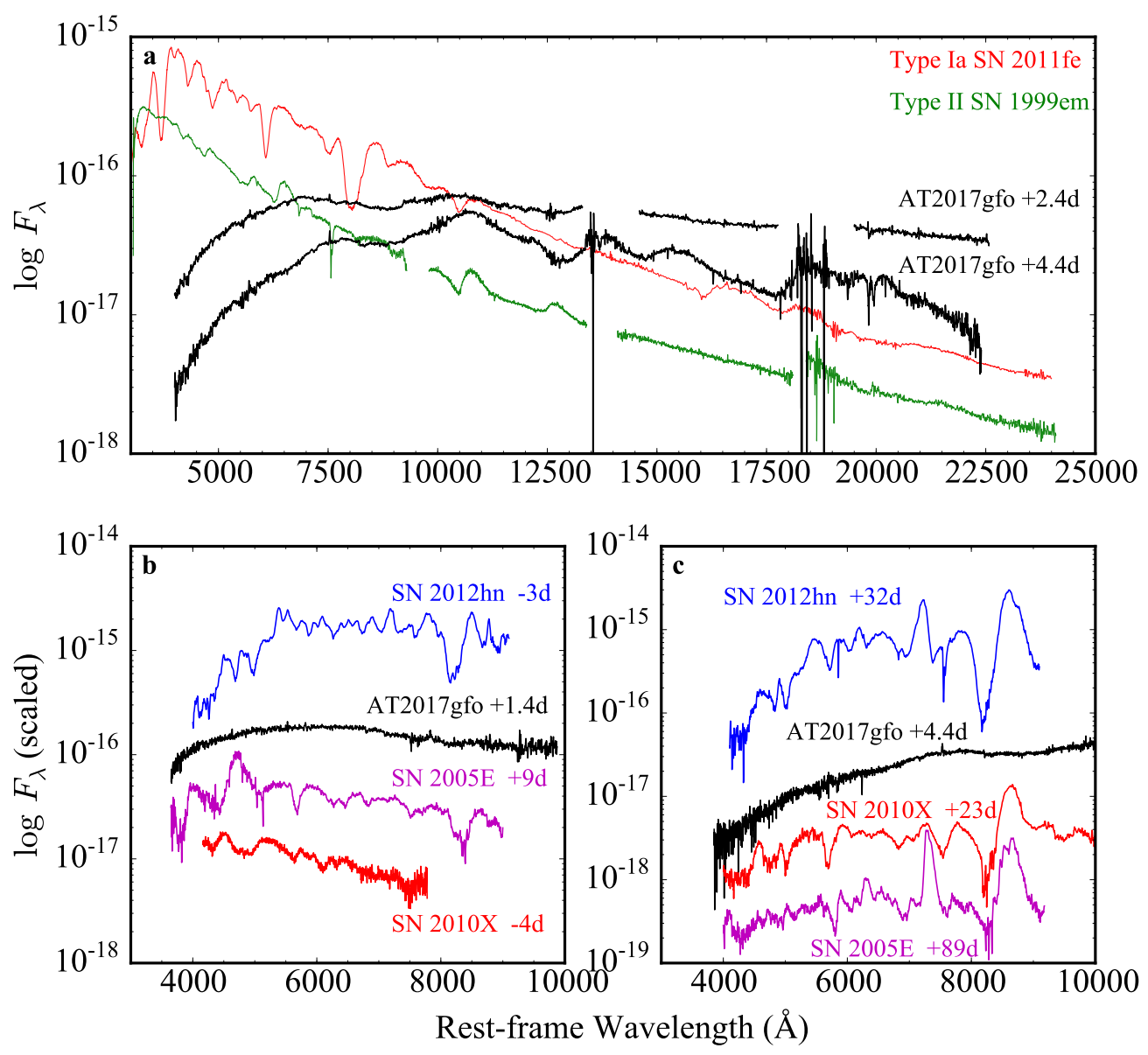

Extended Data Figure 3 | Spectral comparisons a) Comparison of our Xshooter spectra of AT2017gfo with early-time (4 - 5 days post explosion) optical and near infra-red spectra of Type la SN $2011 \mathrm{fe}^{72}$ and Type II-Plateau SN 1999em ${ }^{73}$. The spectra have been scaled for comparison purposes. b) Comparison of our earliest spectrum of AT2017gfo (+1.4 days after explosion) with a sample of Type I events, which share some common properties with AT2017gfo such as faint absolute magnitudes and/or fast evolution and/or explosion environments without obvious star formation. c) Comparison of the +4.4 day spectrum of AT2017gfo with our sample of faint and fast-evolving events at later phases. 

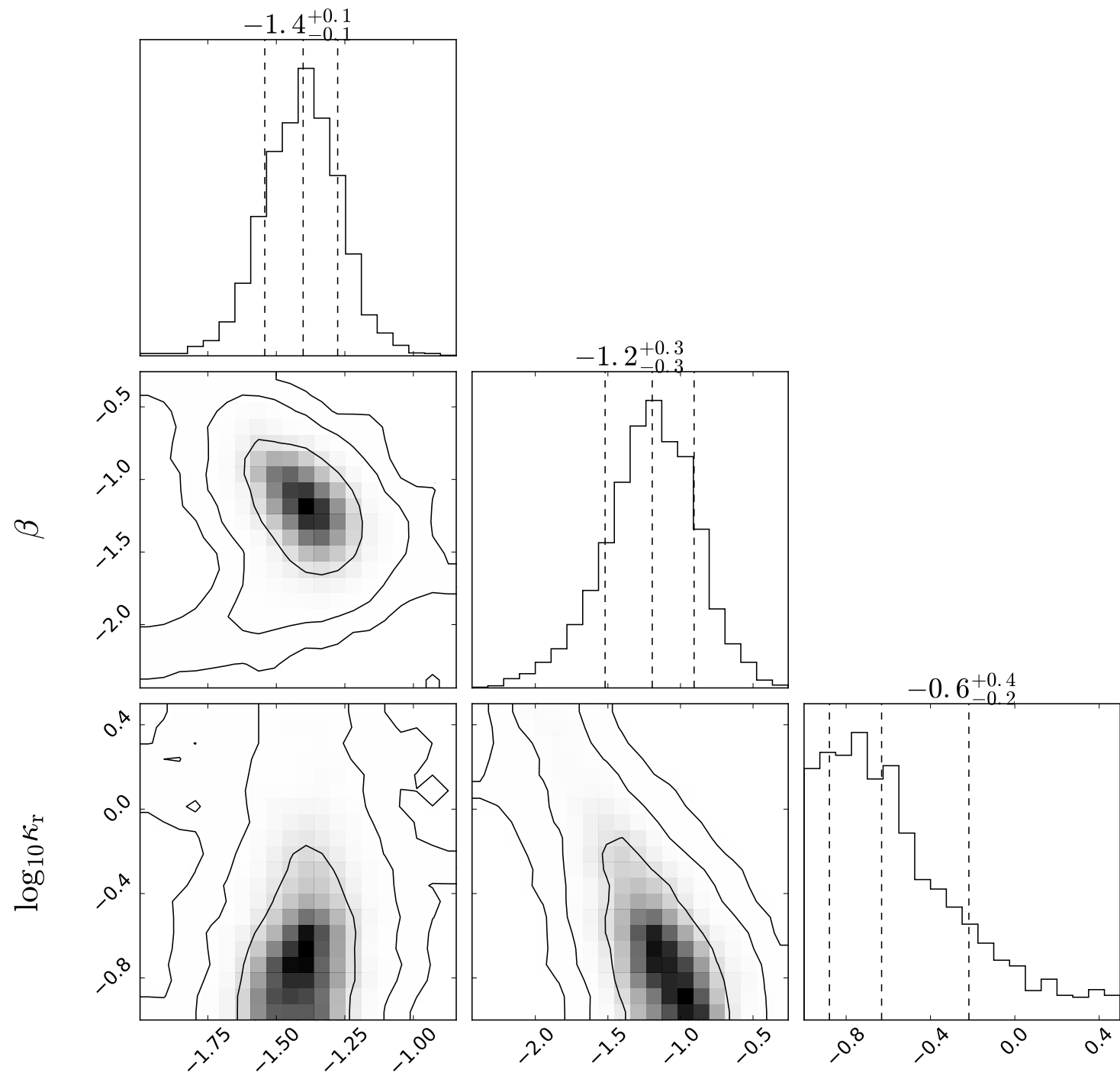

$\log _{10}\left(M_{\mathrm{ej}}\right)$

$\beta$

$\log _{10} \kappa_{\mathrm{r}}$

Extended Data Figure $4 \quad$ Posterior probability plots of our model light curve fits. This is the Arnett formalism which includes a power law term for radioactive powering. We show the $68 \%$ quantile in all plots and $95 \%$ and $99.7 \%$ levels in the $2 \mathrm{D}$ histograms. We quote the maximum posterior fit value and the $68 \%$ quantile range as uncertainty. 

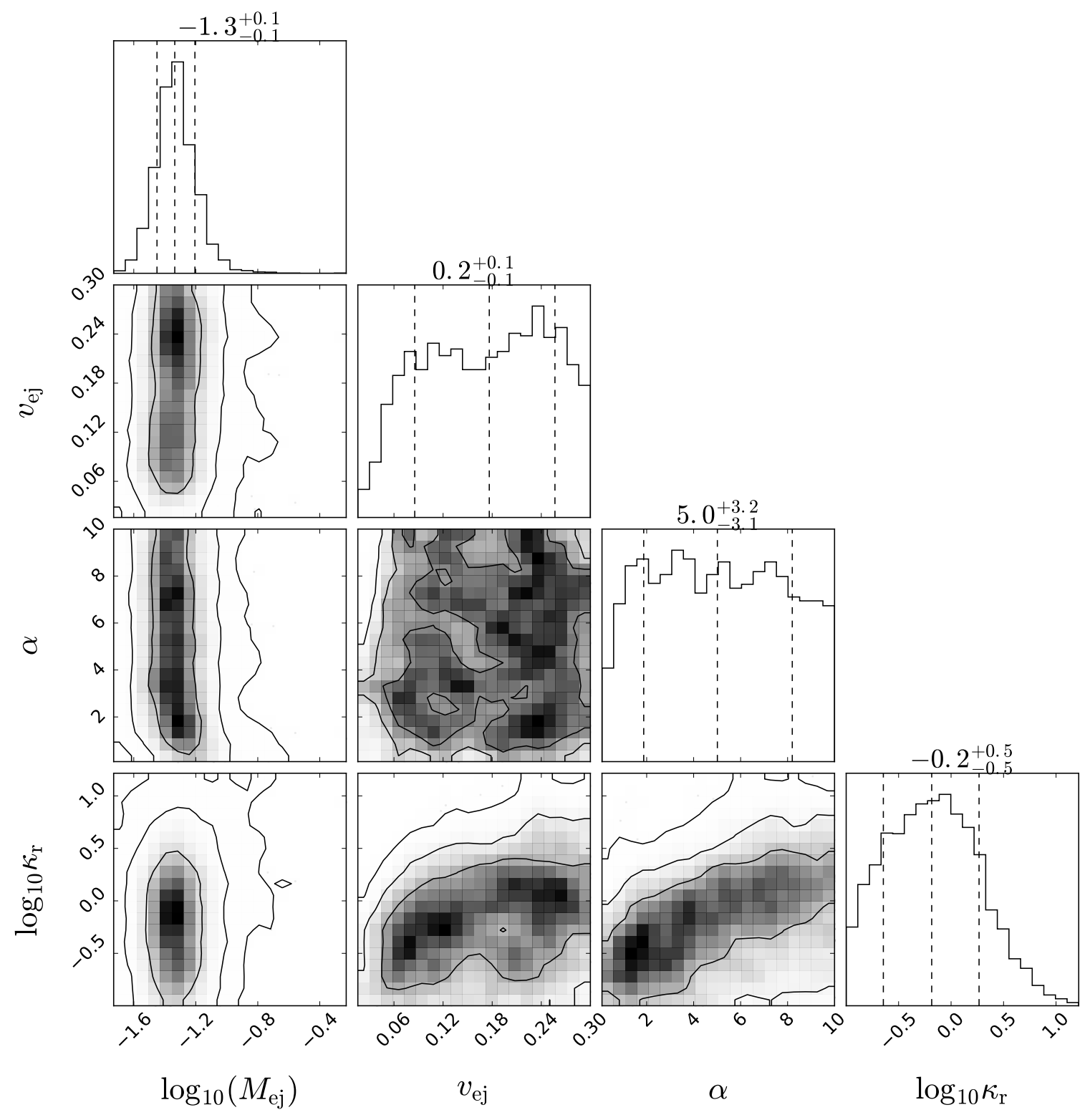

\section{Extended Data Figure $5 \quad$ Posterior probability plots of our model light curve fits for} the parameterised Metzger mode ${ }^{19}$ as described in the main manuscript. As in Fig. 4, we show the $68 \%$ quantile in all plots and $95 \%$ and $99.7 \%$ levels in the $2 \mathrm{D}$ histograms. We quote the maximum posterior fit value and the $68 \%$ quantile range as uncertainty. 


\begin{tabular}{|c|c|c|c|c|c|c|c|}
\hline $\begin{array}{c}\text { Date } \\
\text { (UT) }\end{array}$ & MJD & Phase & Instrument & Grism/Grating & Range & Slit & Resolution \\
\hline $2017-08-18$ & 57983.971662 & +1.44 & NTT+EFOSC2 & Gr\#11+16 & $333-997$ & 1.5 & $1148 / 756$ \\
$2017-08-19$ & 57984.976164 & +2.45 & NTT+EFOSC2 & Gr\#11+16 & $333-997$ & 1.5 & $1148 / 756$ \\
$2017-08-19$ & 57984.978309 & +2.45 & VLT+Xshooter & fixed & $370-2279$ & 1.0 & $70 / 90 / 55$ \\
$2017-08-20$ & 57985.973069 & +3.45 & NTT+EFOSC2 & Gr\#11+16 & $333-997$ & 1.0 & $765 / 504$ \\
$2017-08-21$ & 57986.966396 & +4.44 & NTT+SOFI & Blue Grism & $938-1646$ & 1.0 & 545 \\
$2017-08-21$ & 57986.976138 & +4.45 & VLT+Xshooter & fixed & $370-2279$ & 1.0 & $70 / 90 / 55$ \\
\hline
\end{tabular}

Extended Data Table 1 | Log of spectroscopic observations. The phase is with respect to the LIGO-Virgo detection of 57982.528524. 


\begin{tabular}{|c|c|c|c|c|c|c|c|c|c|c|}
\hline Date & UT & MJD & $\begin{array}{l}\text { Phase } \\
\text { (d) }\end{array}$ & $\begin{array}{c}U \\
\text { (mag) }\end{array}$ & $\begin{array}{c}g \\
\text { (mag) }\end{array}$ & $\begin{array}{c}r \\
\text { (mag) }\end{array}$ & $\begin{array}{c}i \\
\text { (mag) }\end{array}$ & $\begin{array}{c}z \\
\text { (mag) }\end{array}$ & $\begin{array}{c}y \\
\text { (mag) }\end{array}$ & Telescope \\
\hline 2017-08-18 & $05: 33$ & 57983.23125 & 0.696 & ...... & ...... & ...... & $17.24 \pm 0.06$ & $17.26 \pm 0.06$ & $17.38 \pm 0.10$ & PS1 \\
\hline 2017-08-18 & $18: 12$ & 57983.75833 & 1.218 & ...... & ..... & $17.89 \pm 0.03$ & ..... & ...... & ...... & $1.5 \mathrm{~B}$ \\
\hline 2017-08-18 & $23: 15$ & 57983.96875 & 1.427 & ...... & $18.49 \pm 0.04$ & $17.99 \pm 0.01$ & $17.85 \pm 0.05$ & $17.72 \pm 0.03$ & ..... & GROND \\
\hline 2017-08-19 & 01:09 & 57984.04811 & 1.505 & $20.25 \pm 0.29$ & $\ldots .$. & $\ldots$. & $\ldots$. & $\ldots .$. & ...... & NTT \\
\hline 2017-08-19 & $05: 33$ & 57984.23125 & 1.686 & ...... & ...... & ...... & $17.87 \pm 0.06$ & $17.78 \pm 0.07$ & $17.58 \pm 0.11$ & PS1 \\
\hline 2017-08-19 & $18: 16$ & 57984.76111 & 2.211 & ...... & ...... & $18.80 \pm 0.07$ & ..... & ...... & ..... & $1.5 \mathrm{~B}$ \\
\hline 2017-08-19 & $23: 15$ & 57984.96892 & 2.417 & ...... & $20.19 \pm 0.11$ & $19.13 \pm 0.17$ & $18.58 \pm 0.04$ & $18.33 \pm 0.06$ & $\ldots$. & GROND \\
\hline 2017-08-20 & 01:19 & 57985.05497 & 2.502 & $>19.6$ & ...... & $\ldots .$. & ..... & ..... & ..... & NTT \\
\hline 2017-08-20 & $05: 33$ & 57985.23125 & 2.676 & ...... & ...... & ...... & $18.44 \pm 0.09$ & $18.31 \pm 0.07$ & $18.08 \pm 0.11$ & PS1 \\
\hline 2017-08-20 & $18: 38$ & 57985.77639 & 3.216 & ..... & ...... & $19.52 \pm 0.13$ & ...... & ...... & ..... & $1.5 \mathrm{~B}$ \\
\hline 2017-08-20 & $23: 23$ & 57985.97433 & 3.412 & ...... & $21.13 \pm 0.16$ & $19.81 \pm 0.02$ & $19.03 \pm 0.01$ & $18.74 \pm 0.02$ & ...... & GROND \\
\hline 2017-08-21 & 05:39 & 57986.23556 & 3.671 & ..... & ...... & ..... & $>17.8$ & $18.10 \pm 0.30$ & $>17.7$ & PS1 \\
\hline 2017-08-21 & $23: 22$ & 57986.97426 & 4.402 & ..... & $21.58 \pm 0.22$ & $20.53 \pm 0.05$ & $19.51 \pm 0.04$ & $19.07 \pm 0.06$ & ..... & GROND \\
\hline 2017-08-22 & 05:39 & 57987.23556 & 4.661 & ...... & $\ldots$. & $\ldots .$. & $\ldots$. & $>18.8$ & $\ldots$ & PS1 \\
\hline $2017-08-23$ & $05: 36$ & 57988.23354 & 5.649 & ..... & ...... & ..... & ..... & ..... & $18.95 \pm 0.44$ & PS1 \\
\hline 2017-08-24 & $05: 31$ & 57989.23024 & 6.636 & ..... & ...... & ...... & ..... & ..... & $19.31 \pm 0.43$ & PS1 \\
\hline 2017-08-24 & $23: 35$ & 57989.98317 & 7.382 & ..... & $>20.5$ & $>20.6$ & $>20.5$ & $>19.7$ & $\ldots .$. & GROND \\
\hline 2017-08-25 & $05: 30$ & 57990.22962 & 7.626 & $\ldots$. & $\ldots$. & $\ldots$. & $\ldots$. & $\ldots$. & $>18.9$ & PS1 \\
\hline 2017-08-25 & $23: 13$ & 57990.96775 & 8.357 & ..... & $>22.2$ & $>21.7$ & $>21.1$ & $>21.5$ & ...... & GROND \\
\hline 2017-08-25 & $23: 34$ & 57990.97993 & 8.369 & ..... & $>21.0$ & $>21.4$ & $>21.1$ & $>20.4$ & $\ldots$. & NTT \\
\hline 2017-08-26 & $23: 15$ & 57991.96940 & 9.349 & ...... & ref & ref & ref & ref & ...... & GROND \\
\hline
\end{tabular}

Extended Data Table 2 Optical photometric measurements. The UT and MJD are at the start of the exposure. The phase is with respect to the LIGO-Virgo detection of 57982.528524 (rest frame). All magnitudes are in the AB system. The GROND epoch on the 2017-08-26 was used as the reference template for image subtraction for all GROND epochs up to this date. All limits are $3 \sigma$. 


\begin{tabular}{|c|c|c|c|c|c|c|c|}
\hline Date & UT & MJD & $\begin{array}{c}\text { Phase } \\
\text { (d) }\end{array}$ & $\begin{array}{c}J \\
(\mathrm{mag})\end{array}$ & $\begin{array}{c}H \\
\text { (mag) }\end{array}$ & $\begin{array}{c}K_{s} \\
(\mathrm{mag})\end{array}$ & Telescope \\
\hline $2017-08-18$ & $23: 15$ & 57983.96875 & 1.427 & $17.58 \pm 0.07$ & $17.64 \pm 0.08$ & $18.14 \pm 0.15$ & GROND \\
\hline 2017-08-19 & $23: 15$ & 57984.96892 & 2.417 & $17.73 \pm 0.09$ & $17.64 \pm 0.08$ & $17.90 \pm 0.10$ & GROND \\
\hline $2017-08-20$ & $23: 23$ & 57985.97433 & 3.413 & $17.95 \pm 0.07$ & $17.72 \pm 0.07$ & $17.86 \pm 0.10$ & GROND \\
\hline $2017-08-21$ & $23: 22$ & 57986.97426 & 4.403 & $18.17 \pm 0.07$ & $18.02 \pm 0.10$ & $17.74 \pm 0.11$ & GROND \\
\hline 2017-08-24 & $23: 35$ & 57989.98317 & 7.383 & $19.26 \pm 0.28$ & $18.74 \pm 0.06$ & $18.40 \pm 0.12$ & GROND \\
\hline $2017-08-25$ & $23: 13$ & 57990.96775 & 8.358 & $19.64 \pm 0.11$ & $19.26 \pm 0.26$ & $18.86 \pm 0.16$ & GROND \\
\hline $2017-08-26$ & $23: 15$ & 57991.96940 & 9.350 & $20.23 \pm 0.10$ & $19.66 \pm 0.14$ & $19.03 \pm 0.20$ & GROND \\
\hline $2017-08-27$ & $23: 24$ & 57992.97527 & 10.346 & $21.02 \pm 0.22$ & ..... & ..... & NTT \\
\hline $2017-08-28$ & $00: 22$ & 57993.01593 & 10.386 & $>21.2$ & $20.17 \pm 0.34$ & $19.50 \pm 0.22$ & GROND \\
\hline $2017-08-28$ & 23:03 & 57993.96019 & 11.322 & $\ldots .$. & $20.05 \pm 0.20$ & $\ldots \ldots$ & NTT \\
\hline $2017-08-28$ & $23: 22$ & 57993.97428 & 11.335 & $>21.3$ & $>19.3$ & $19.64 \pm 0.30$ & GROND \\
\hline $2017-08-29$ & $22: 56$ & 57994.95526 & 12.307 & ...... & ..... & $19.40 \pm 0.14$ & NTT \\
\hline 2017-08-29 & $23: 49$ & 57994.97369 & 12.324 & ref & ref & ref & GROND \\
\hline $2017-08-30$ & 23:03 & 57995.96075 & 13.303 & ...... & ...... & $19.67 \pm 0.20$ & NTT \\
\hline 2017-09-02 & $23: 12$ & 57998.96696 & 16.280 & ...... & $\ldots \ldots$ & $>17.9$ & NTT \\
\hline 2017-09-03 & $23: 18$ & 57999.97074 & 17.274 & ...... & ...... & $>20.2$ & NTT \\
\hline 2017-09-04 & $23: 12$ & 58000.96635 & 18.260 & ...... & ..... & $20.76 \pm 0.35$ & NTT \\
\hline
\end{tabular}

Extended Data Table 3 | Near infra-red photometric measurements. The UT and MJD are at the start of the exposure. The phase is with respect to the LIGO-Virgo detection of 57982.528524 (rest frame). All magnitudes are in the AB system. The GROND epoch on the 2017-08-29 was used as the reference template for image subtraction for all GROND epochs up to this date. All limits are $3 \sigma$. 


\begin{tabular}{|c|c|c|c|c|}
\hline MJD & Phase (days) & $L_{\mathrm{Bol}}$ & $T_{\mathrm{BB}}(\mathrm{K})$ & $R_{\mathrm{BB}}(\mathrm{cm})$ \\
\hline 57983.167 & 0.638 & $42.051 \pm 0.123$ & $7600 \pm 2000$ & $6.878 \pm 1.912 \times 10^{14}$ \\
57983.231 & 0.696 & $41.960 \pm 0.115$ & $7500 \pm 1800$ & $6.360 \pm 1.638 \times 10^{14}$ \\
57983.563 & 1.033 & $41.901 \pm 0.122$ & $7300 \pm 1500$ & $6.273 \pm 1.305 \times 10^{14}$ \\
57983.969 & 1.427 & $41.693 \pm 0.084$ & $5950 \pm 500$ & $7.431 \pm 0.465 \times 10^{14}$ \\
57984.969 & 2.417 & $41.348 \pm 0.093$ & $3800 \pm 300$ & $1.225 \pm 0.054 \times 10^{15}$ \\
57985.974 & 3.413 & $41.211 \pm 0.081$ & $3000 \pm 200$ & $1.678 \pm 0.059 \times 10^{15}$ \\
57986.974 & 4.403 & $41.107 \pm 0.101$ & $2900 \pm 150$ & $1.593 \pm 0.025 \times 10^{15}$ \\
57989.983 & 7.383 & $40.808 \pm 0.208$ & $2200 \pm 500$ & $1.962 \pm 0.307 \times 10^{15}$ \\
57990.968 & 8.358 & $40.649 \pm 0.215$ & $2400 \pm 600$ & $1.373 \pm 0.248 \times 10^{15}$ \\
57991.969 & 9.350 & $40.177 \pm 0.196$ & $1900 \pm 400$ & $1.272 \pm 0.184 \times 10^{15}$ \\
57993.016 & 10.386 & $39.939 \pm 0.316$ & $1900 \pm 500$ & $9.673 \pm 0.950 \times 10^{14}$ \\
57993.974 & 11.335 & $39.921 \pm 0.371$ & $1900 \pm 350$ & $9.475 \pm 0.882 \times 10^{14}$ \\
57994.955 & 12.307 & $39.880 \pm 0.265$ & $1900 \pm 500$ & $9.038 \pm 1.353 \times 10^{14}$ \\
57995.961 & 13.303 & $39.766 \pm 0.407$ & $1900 \pm 500$ & $7.926 \pm 0.011 \times 10^{14}$ \\
\hline
\end{tabular}

Extended Data Table 4 | Bolometric light curve, temperature and radius evolution. These values were calculated as described in the Methods section, $L_{\mathrm{bol}}$ is in erg s $\mathrm{s}^{-1}$. The two points at 0.638 and 1.033 days were estimated from the Swift data 5960 . The phase is with respect to the LIGO-Virgo detection of 57982.528524 (rest frame). 